\title{
Excitation of Crossflow Instabilities in a Swept Wing Boundary Layer
}

\author{
Mark H. Carpenter*, Meelan Choudhari ${ }^{\dagger}, \mathrm{Fei} \mathrm{Li}^{\ddagger}$, Craig L. Streett ${ }^{\S}$, Chau-Lyan Chang $₫$ \\ NASA Langley Research Center, Hampton, VA 23681, USA
}

\begin{abstract}
The problem of crossflow receptivity is considered in the context of a canonical 3D boundary layer (viz., the swept Hiemenz boundary layer) and a swept airfoil used recently in the SWIFT flight experiment performed at Texas A\&M University. First, Hiemenz flow is used to analyze localized receptivity due to a spanwise periodic array of small amplitude roughness elements, with the goal of quantifying the effects of array size and location. Excitation of crossflow modes via nonlocalized but deterministic distribution of surface nonuniformity is also considered and contrasted with roughness induced acoustic excitation of Tollmien-Schlichting waves. Finally, roughness measurements on the SWIFT model are used to model the effects of random, spatially distributed roughness of sufficiently small amplitude with the eventual goal of enabling predictions of initial crossflow disturbance amplitudes as functions of surface roughness parameters.
\end{abstract}

\section{Introduction}

Surface roughness characteristics are known to exert profound influence on the onset of transition in swept wing boundary layers. A striking example of surface quality effects on transition is documented in Ref. 1, wherein transition is noted to move toward the trailing edge from approximately 25 to 30 percent chord to 80 percent chord when the painted leading edge was polished to reduce the roughness amplitude from $1.0 \mu \mathrm{m}$ root mean square (RMS) $(3.8 \mu \mathrm{m}$ average peak-to-peak) to $0.3 \mu \mathrm{m}$ RMS $(2.2 \mu \mathrm{m}$ average peak-to-peak). Had linear stability based $\mathrm{N}$-factor methods been applied to predict the onset of transition in this case, the correlating N-factor would have increased from 8 to 16. Similarly large N-factor values have been reported in the context of earlier investigations of swept wing boundary layers at ONERA. ${ }^{2}$ Because the small amplitude surface roughness primarily seeds the stationary crossflow instabilities that eventually lead to transition, prediction uncertainties of this magnitude can only be minimized by accounting for the receptivity phase in the transition prediction process. Inclusion of receptivity is particularly critical in the context of laminar flow control via distributed roughness elements (DREs), ${ }^{3}$ since the delay in transition is achieved via controlled seeding of less unstable crossflow modes and the application of DRE heights outside of an optimal range may result in inadequate control at one end (i.e., too small heights) and premature transition (or over-control) at the other end. The latter possibility is particularly likely for high Reynolds number configurations as the linear amplification ratios achieve substantial magnitudes across a broad range of stationary crossflow wavelengths. ${ }^{4}$

Roughness induced excitation of crossflow instabilities was examined in Ref. 5, wherein preferential excitation of stationary crossflow vortices was confirmed on the basis of a straightforward extension of the Goldstein $^{6}-$ Ruban $^{7}$ theory. Potentially substantial effects of surface curvature and mean-flow nonparallelism were pointed out in Ref. 8 and the effects of nonlinear roughness height were recently addressed in Ref. 9. References ${ }^{8,10-19}$ describe the various applicable prediction methodologies (ranging from the inhomogeneous form of linear stability equations, parabolized stability equations, and linearized or full Navier-Stokes equations) and their application to a broader class of flows. Much of this work, however, pertains to a single,

\footnotetext{
*Corresponding author. E-mail: mark.h.carpenter@nasa.gov.

$\dagger$ E-mail: meelan.choudhari@nasa.gov, Associate Fellow AIAA.

${ }^{\ddagger} \mathrm{E}$-mail: fei.li@nasa.gov.

$\S$ E-mail: craig.l.streett@nasa.gov, Member AIAA.

『E-mail: chau.lyan.chang@nasa.gov, Member AIAA.
} 
spanwise periodic array of roughness elements, while studies reporting the receptivity to spatially nonlocalized roughness distributions are less common. (See Ref. 20 for a theoretical study of nonlocalized roughness effects or Ref. 21 for an experimental investigation on the subject.) For small amplitude roughness, at least, the theoretical framework for the crossflow receptivity to distributed surface roughness is similar to that of the acoustic excitation of Tollmien-Schlichting waves via nonlocalized roughness. ${ }^{10}$ Yet, this framework needs to be applied with due consideration to the effect of surface curvature and mean boundary layer growth on both receptivity and stability characteristics of stationary crossflow modes.

An integrated approach combining receptivity with linear and nonlinear growth of the stationary crossflow instability was demonstrated in Ref. 19, using parabolized stability equations (PSE) and adjoint PSE as implemented in the Langley Stability \& Transition Analysis Code (LASTRAC). This combination of tools enables efficient and accurate prediction of disturbance evolution in many cases of interest. However, when the disturbance wavelength of interest changes rapidly in space, the basic parabolizing assumption becomes suspect. There, the full elliptic nature of the flow must be included to achieve an accurate disturbance prediction. Harmonic Linear Navier-Stokes (HLNS) tools have been shown to be successful in complementing existing PSE tools in these flow environments. (See the work of Streett ${ }^{13}$ for a detailed discussion of the HLNS approach.) The increased fidelity of the HLNS approach does have its drawbacks: most notably the increased computational costs, which can exceed comparable PSE simulation times by at least an order of magnitude. Thus, the HLNS approach is relatively less suitable for performing extensive design investigations or optimization studies.

This paper addresses two fundamental challenges. First, a discrete adjoint formulation is introduced to complement an existing Harmonic Linear Navier-Stokes capability. Profound improvements in efficiency are achieved with the new adjoint approach as shown in numerous examples. Furthermore, it is shown that extensive design and optimization studies are now straightforward using the higher fidelity HLNS equations. With the new tool in hand, we consider both localized and distributed receptivity mechanisms for crossflow vortex instability. Two different swept-airfoil configurations are examined as part of the parameter study. First, we consider a canonical 3D boundary layer (viz., the swept Hiemenz boundary layer), followed by a realistic swept airfoil geometry employed during a recent SWIFT experiment performed at Texas A\&M University. ${ }^{1,22}$ Localized receptivity due to a spanwise periodic array of small amplitude roughness elements is first analyzed using Hiemenz flow, with the goal of quantifying the effects of array location and element size. Next, the excitation of crossflow modes via a nonlocalized but deterministic distribution of surface nonuniformity is considered, and contrasted with the spanwise periodic roughness elements as well as roughness induced acoustic excitation of Tollmien-Schlichting waves. Finally, the SWIFT experimental flow conditions are used to study the effects of random, spatially distributed roughness (of sufficiently small amplitude). The eventual goal is to predict the initial crossflow amplitudes via a reduced order model of the roughness geometry, using only a small number of amplitude and texture parameters to represent the surface roughness. It is envisioned that this study will help identify the most relevant parameters and, hence, contribute to the potential development of an appropriate database pertaining to the natural roughness of typical aerodynamic surfaces. ${ }^{17}$

The paper is organized as follows. Section II gives a general description of the SWIFT flight experiment. Section III(a) summarizes the existing HLNS formulation originally developed by Streett, ${ }^{13}$ while Section III(b) introduces a complementary adjoint formulation. Section IV includes a brief description of the theoretical aspects of receptivity. Section V presents results. First, a detailed study of the well known incompressible swept Hiemenz flow is presented, including extensive validation of the new adjoint approach. Next, the adjoint approach is utilized to compare and contrast the receptivity of the boundary layer to spanwise periodic roughness elements, and deterministic nonlocalized roughness distributions. Finally, a detailed study of the receptivity over the SWIFT airfoil configuration is presented, beginning first with a description of the surface characteristics of the wing. Next, a parametric study of optimal roughness element size and location is presented. Finally, the effects of random spatially distributed roughness of sufficiently small amplitude is presented. Section VI summarizes the work.

\section{Experimental Setup}

The flow configuration of interest in the present paper corresponds to the SWIFT flight experiment conducted at Texas A\&M University. ${ }^{1,22}$ A 30-degree swept airfoil test article was mounted vertically beneath the wing of a Cessna O-2A Skymaster aircraft and flown at chord Reynolds numbers of 7 to 8 
million, angle of attack $\alpha_{A o A}=-4.7^{\circ}$, and airspeed 180.0 KTAS (knots true air speed), to investigate the feasibility of discrete roughness elements (DRE) technology for SensorCraft applications. The goal was to achieve a minimum of $60 \%$ laminar flow over the suction side of the model by appropriately placed DREs.

For the sake of clarity, a single DRE is simply a bump (or, alternatively a dimple) on the surface of the airfoil, e.g., in the form of a shallow cylindrical disk (a hockey puck). A spanwise, periodic array of such elements is constructed by aligning multiple elements parallel to the spanwise direction, separated by a distance $d$. Thus, the array is periodic with length $d$. Spanwise-periodic DREs provide a passive means of controlling the crossflow instability responsible for transition over a swept wing without the need for an active device (e.g. suction). Indeed, Saric et al. ${ }^{3}$ demonstrated that roughness elements on the scale of micro-meters were effective in moving the transition location. The method for spanwise spacing of DREs is outlined in the work of Reibert et al., ${ }^{23}$ whereby appropriately forced modes distort the mean flow to inhibit the naturally occurring most unstable crossflow vortices, thus delaying transition. Conversely, DREs with different placement and character can be used to excite the unstable modes thus forcing transition and achieving a large chordwise extent of turbulent flow.

Linear stability theory applied to the SWIFT configuration identified the $4.5 \mathrm{~mm}$ wavelength crossflow mode as being extremely unstable, whereby a DRE spanwise spacing corresponding to the more stable $2.25 \mathrm{~mm}$ mode could be used for transition control via DREs. The placement of the DREs is typically near the neutral point of the control $(2.25 \mathrm{~mm})$ mode; i.e. between $1 \%$ and $2 \%$ chord for the inboard and outboard stations, respectively.

For the work described in this paper, the mean flow is computed with a boundary layer solver based on the infinite span approximation, using the surface pressure distribution derived from the CFD solution. ${ }^{22}$ Spanwise variations in the computed pressure distribution over the mid-span segment of the suction surface were relatively small, so that the region between the rows of static pressure ports at 13 inch and 29 inch span locations outboard of the mounting plane are assumed to approximate (locally) infinite span conditions. Transition measurements, both without and with the use of DREs, were reported in Ref. ${ }^{22}$ High-fidelity computational analysis of the DRE based control was reported in Ref. 24.

\section{Analysis Codes}

Many of the receptivity results presented herein are obtained using a harmonic variant of the linearized incompressible Navier-Stokes equations. The basic methodology and algorithm was reported in Ref. ${ }^{13}$ An adjoint formulation has been developed to complement and expedite the original algorithm. A brief outline of each approach is now presented. (See Refs. ${ }^{13}$ or $^{25}$ for further details.)

\section{III.A. Incompressible Harmonic Linearized Navier-Stokes}

The flows of interest in this study (i.e. $M_{\infty} \leq 0.25$ ) are well represented using the incompressible NavierStokes (NS) equations. The derivation of the harmonic, linearized incompressible NS equations begins with an expansion of the flow around a base state:

$$
\mathbf{U}(x, y, z, t)=\overline{\mathbf{U}}(x, y, z)+\epsilon \mathbf{u}(x, y, z, t) .
$$

where $\mathbf{U}=[P, U, V, W]^{T}, \overline{\mathbf{U}}=[\bar{P}, \bar{U}, \bar{V}, \bar{W}]^{T}, \mathbf{u}=[p, u, v, w]^{T}$ and the components $[p, u, v, w]$ are the incompressible pseudo pressure- and $(x, y, z)$ velocity-perturbations in the $x, y$ and $z$ coordinate directions, respectively. By construction, the base state $\overline{\mathbf{U}}$ satisfies the steady incompressible NS equations, or a suitable approximate form; e.g., the boundary-layer equations or the Falkner-Skan-Cooke similarity equations. Substituting equation (1) into the nonlinear incompressible NS equations, and collecting terms of order $\epsilon$, yields a linear set of disturbance equations in the variables $\mathbf{u}(x, y, z, t ; \overline{\mathbf{U}}){ }^{\text {a }}$

The linear disturbance equation for the variables $\mathbf{u}(x, y, z, t ; \overline{\mathbf{U}})$ is further simplified by the ansatz

$$
\frac{\partial \mathbf{u}}{\partial t}=-i \omega \mathbf{u} \quad ; \quad \frac{\partial \mathbf{u}}{\partial z}=+i \beta \mathbf{u}
$$

The first ansatz limits solutions to a single harmonic, specified by the frequency $\omega$, while the second assumes spanwise periodicity in $z$ at a specified wavenumber $\beta$. Both approximations are well suited for the infinite

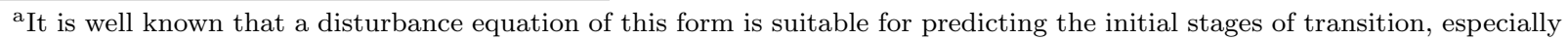
when amplitudes are orders of magnitude smaller than the background base flow $\overline{\mathbf{U}}$.
} 
span swept wing flows studied herein. The time-harmonic and spanwise periodicity assumptions reduce the 4-dimensional original formulation for the variables $\mathbf{u}(x, y, z, t ; \overline{\mathbf{U}})$, to one involving only two spatial dimensions: for the variables $\tilde{\mathbf{u}}(x, y ; \omega, \beta, \overline{\mathbf{U}})$.

$$
\begin{array}{r}
d(x, y ; \omega, \beta, \overline{\mathbf{U}}) \tilde{\mathbf{u}}+a_{x}(x, y ; \beta, \overline{\mathbf{U}}) \frac{\partial \tilde{\mathbf{u}}}{\partial x}+a_{y}(x, y ; \beta, \overline{\mathbf{U}}) \frac{\partial \tilde{\mathbf{u}}}{\partial y}= \\
\quad b_{x x}(x, y ; \overline{\mathbf{U}}) \frac{\partial^{2} \tilde{\mathbf{u}}}{\partial x^{2}}+b_{x y}(x, y ; \overline{\mathbf{U}}) \frac{\partial^{2} \tilde{\mathbf{u}}}{\partial x \partial y}+b_{y y}(x, y ; \overline{\mathbf{U}}) \frac{\partial^{2} \tilde{u}}{\partial y^{2}}
\end{array}
$$

Remark: While equation (3) is expressed in terms of the independent variables $(x, y)$, it could equally well represent an orthogonal coordinate frame $\left(x_{1}, x_{2}\right)$. Here, the independent variables $(x, y)$ correspond to the orthogonal, streamwise and wall-normal directions.

Boundary conditions are a sensitive aspect of this formulation contributing both to physical well-posedness (accuracy) as well as numerical conditioning. Typically, Dirichlet and/or Neumann conditions are specified on the inflow, wall and farfield boundaries. The specification of the down-stream boundary condition requires a different approach, however. A buffer-domain technique, ${ }^{26}$ in which the momentum equations are smoothly parabolized to convect disturbances through the outflow boundary, was found to be robust and reliable alternative to conventional Dirichlet or Neumann conditions.

Solution of the partial differential equations (PDEs) given in equation (3) requires the spatial derivatives to be discretized, resulting in a linear matrix equation of the form

$$
A_{H L N S} \mathbf{w}=\mathbf{f}
$$

where $\mathbf{w}$ is the solution vector for all discretely orthogonal points $\left(y_{j}, x_{i}\right)$ in the field, and the right-hand side vector $\mathbf{f}$ contains boundary conditions (e.g. wall blowing/suction, or free-stream disturbances).

The structure of the matrix $A_{H L N S}$ depends on the form of the discrete spatial derivative operators. In the present study, the streamwise direction $x_{i}$ is discretized using a variable stepsize fourth-order centered difference technique, while the wall normal direction $y_{j}$ uses a mapped Chebyshev collocation technique. Grid refinement studies were performed in all cases, and established that all results were accurate to approximately three significant digits. The maximum tensor product mesh needed was of dimension $4,000 \times 71$, while most required significantly fewer points (i.e. $1,500 \times 50$ ).

This discretization results in a block pentadiagonal matrix if a lexigraphic $(j, i)$ ordering is used. The block size $N_{b}$ is given by $N_{b}=4 * n y$ where $n y$ is the number of $y_{j}$ Chebyshev collocation points. A direct solver was used in all cases to invert the linear system. The single-processor CPU times (Intel Xeon operating at $3.0 \mathrm{GHz}$ ) were in the range $1-10$ minutes depending on the particular grid. Inversion times scaled linearly with the streamwise dimension $x_{i}$ and cubically with the wall-normal $y_{j}$ dimension. The problems considered in this work were generally too small to benefit appreciably from multi-threaded parallel approaches (e.g. OpenMP BLAS2 and BLAS3 operations). A modest improvement was achieved by splitting the streamwise direction into multiple blocks, coupled using a Schur-Complement approach. This technique enables the use of multiple cores on a dual socket quad-core workstation and leads to a reduction in wall-clock time by a factor $2-3$ when using all 8 cores.

Summarizing, simulations using the quasi-3D harmonic equations are easily preformed on commodity hardware (\$1000), thus allowing "routine" engineering calculations in many setting even without any adjoint formulation.

\section{III.B. Adjoint formulation}

Although a continuous adjoint approach was adopted in previous PSE work, ${ }^{25}$ herein a discrete adjoint formulation is implemented to expedite the solution of equation (3). A brief summary of the contributing factors that led to the choice of the discrete approach is now presented.

\section{III.B.1. Background}

There is a long history of the use of adjoint equations in fluid dynamics. Pironeau, ${ }^{27-29}$ first used the adjoint approach for design studies followed shortly thereafter by design optimization work in CFD (potential/Euler/NS) by Jameson. ${ }^{30-32}$ The breadth and complexity of applications and maturity of the field progressed dramatically over the next decade. ${ }^{33-39}$ 
Adjoint methods are typically divided into two different categories: discrete and continuous adjoint methods, with distinct advantages to each approach. Consider the linear differential equation $\mathcal{L}$ subject to well-posed boundary data $\mathcal{B},{ }^{\text {b }}$

$$
\mathcal{L} u=\left.f_{i}\right|_{\Omega} \quad ; \quad \mathcal{B} u=\left.f_{b}\right|_{\partial \Omega}
$$

and an output cost functional $\mathcal{J}$ of interest

$$
\mathcal{J}=(g, u)_{\Omega}+(h, \mathcal{C} u)_{\partial \Omega} .
$$

Here, the nomenclature (.,.) denotes any standard inner product, while $\Omega$ and $\partial \Omega$ denote action on the volume and boundary, respectively.

The continuous approach seeks a dual formulation

$$
\mathcal{L}^{*} \psi=\left.g_{i}\right|_{\Omega} \quad ; \quad \mathcal{B}^{*} \psi=\left.h_{b}\right|_{\partial \Omega}
$$

where the adjoint equation $\mathcal{L}^{*}$ and adjoint variable $\psi$ satisfy the integral constraint

$$
\left(\mathcal{L}^{*} \psi, u\right)_{\Omega}+\left(\mathcal{B}^{*} \psi, \mathcal{C} u\right)_{\partial \Omega}=(\psi, \mathcal{L} u)_{\Omega}+\left(\mathcal{C}^{*} \psi, \mathcal{B} u\right)_{\partial \Omega}
$$

which is obtained from the original problem using integration by parts (and demanding sufficient smoothness of all quantities that are differentiated). With the dual formulation, the equivalent output cost function becomes

$$
\mathcal{J}=\left(\psi, f_{i}\right)_{\Omega}+\left(\mathcal{C}^{*} \psi, f_{b}\right)_{\partial \Omega} .
$$

The advantage of the adjoint formulation can be profound when the problem of interest requires multiple solutions of equation (4) subject to the datasets $\left[f_{i}^{k}, f_{b}^{k} ; k=1, \cdots\right]$. Rather than one solution with each dataset $k=1, \cdots$, only a single solution of the dual formulation given in equation (6) is required, followed by repeated evaluation of the dual cost function given by equation (8).

Remark. In the continuous approach the adjoint equations and boundary conditions have a precise physical interpretation, and are amenable to mathematical analysis. For example, Giles et al. ${ }^{40}$ derive analytic well-posed adjoint boundary conditions for the Euler equations, and analytically study the behavior of these equations in the vicinity of sonic points, a more difficult proposition when using a discrete approach.

Remark. Note that in the continuous approach the discretization of the equations is explicitly performed on the dual equations given in (6) and (8). The discretized continuous adjoint equations (i.e. the matrix) is not discretely adjoint to anything.

In the discrete adjoint approach, the governing equations given in (3) are first discretized, the result being a matrix problem of the form $\mathbf{A} \mathbf{U}=\mathbf{F}$. A mapping is then sought to transfer the original problem over to an equivalent dual problem. The discrete adjoint is derived as follows. Given a discrete inner product (.,.) defined by the vector product

$$
(\xi, \eta)=\xi^{T} \eta
$$

then the original formulation

$$
\mathbf{A U}=\mathbf{F} \rightarrow \mathbf{I}=(\mathbf{G}, \mathbf{U})
$$

can be expressed using an equivalent dual formulation

$$
\mathbf{A}^{\mathbf{T}} \mathbf{\Psi}=\mathbf{G} \quad \rightarrow \quad \mathbf{I}=(\mathbf{\Psi}, \mathbf{F}) .
$$

A proof of the equivalence of the two problems follows immediately

$$
(\Psi, F)=(\Psi, \mathbf{A} \mathbf{U})=\left(\mathbf{A}^{\mathbf{T}} \mathbf{\Psi}, \mathbf{U}\right)=(\mathbf{G}, \mathbf{U}) .
$$

Note that equation (10) provides the discrete counterpart of the discretized versions of equations (6) and (8). They are not precisely equivalent because the continuous operations of differentiation and integration by parts are not in general exactly satisfied at the discrete level. Thus, results obtained by using the two approaches may not be identical, the differences being at the level of the truncation error in the discretizations.

Remark. The physical significance of the discrete equations is more difficult to interpret. At the very least however, the adjoint solution provides the sensitivity/influence of an arbitrary source term $F$ on the cost functional $I$ of interest.

\footnotetext{
${ }^{\mathrm{b}}$ See Giles et. al. ${ }^{36,37}$ for further details.
} 


\section{III.B.2. Adjoint Boundary Conditions}

The decision to implement a discrete adjoint formulation was strongly motivated by three factors:

- Boundary conditions. A problematic element of the continuous adjoint approach is resolving the complexities brought about by the imposition of buffer domain boundary conditions. Despite the availability of well-posed Dirichlet and Neumann adjoint boundary conditions for the continuous problem (see Giles ${ }^{40}$ ), no such conditions exist for the non-reflecting boundary conditions used at the outflow plane. Furthermore, the buffer domain approach requires modification of the governing equations in the vicinity of the boundary. All these factors must be considered in the derivation of suitable adjoint boundary conditions, not an insurmountable task, but subtle nevertheless.

- Full consistency. The inviscid characteristics terms in the continuous adjoint equations are of opposite sign compared with the HLNS equations. Thus, the adjoint outflow boundary coincides with the inflow boundary of the continuous equations. Thus, by symmetry the adjoint equations require a nonreflecting boundary condition at the upstream boundary, including a modification of the governing equations near that boundary. This introduces an inconsistency between the primal and dual equations at each end of the domain, unless precise boundary conditions were available.

- Coding simplicity. The discrete approach only required building the primal matrix $A_{H L N S}$ followed by its transposition. Everything is accomplished strictly at the level of linear algebra. Furthermore, the primal and dual problems are completely consistent because the same governing equations (including buffer domain) are used for both.

\section{III.B.3. Cost Functionals}

The solution of the incompressible Harmonic Linear Navier-Stokes equations in cases dominated by crossflow instability mechanisms is characterized by a growing streamwise disturbance. The amplitude of the disturbance (at a fixed location) is a direct measure of the efficacy of various forcing mechanism $F$. Cost functionals that are synonymous with the local nature of the disturbance, provide effective sensitivity measures. Indeed, recall that the adjoint solution provides the sensitivity/influence of an arbitrary source term $F$ on the cost functional $I$ of interest. Several cost functionals were tested, and all were found to give qualitatively similar results. Two were thoroughly tested and then used throughout all studies.

The first is the integral of the disturbance "energy" at a fixed streamwise location

$$
I_{u}=\int_{0}^{y \max }\left[u\left(y, x_{i}\right)+v\left(y, x_{i}\right)+w\left(y, x_{i}\right)\right] d y .
$$

where ymax is the outer boundary in the wall normal direction. The second was simply the wall pressure at a fixed streamwise location, $x_{i}$

$$
I_{P}=p\left(0, x_{i}\right) p^{*}\left(0, x_{i}\right) .
$$

Note that both discrete cost functionals $I_{U}$ and $I_{P}$ are essentially "delta functions", being defined at only one streamwise gridpoint, which violates the necessary smoothness constraints needed during the derivation of the continuous adjoint. Qualitatively similar results were obtained when smooth cost functions were used to replace the the point functionals. Comparison of the discrete adjoint from grids with different resolutions requires a normalization step where the discrete adjoint is equated to its continuous counterpart by scaling it with a grid Jacobian.

\section{Theoretical Receptivity}

The basic framework for predicting the roughness induced excitation of stationary crossflow modes is already available, at least for cases where the roughness amplitude is sufficiently small and the wavenumber spectrum of the surface height perturbations overlaps with the length scales of the crossflow vortex instabilities. Based on an extension of the Goldstein ${ }^{6}$ Ruban $^{7}$ theory for localized receptivity, the perturbation quantities associated with an arbitrary crossflow mode of spanwise wavenumber $\beta$ can, in general, be expressed in the form: ${ }^{10}$

$$
q(x, y, z ; \beta)=\hat{C}(x, \beta) \tilde{\phi}(x, y) \exp \left(i \int_{x 0}^{x} \alpha d \xi+\beta z\right)
$$


where $(x, y, z)$ denote the surface tangential, surface normal, and spanwise coordinates within an orthogonal, body fitted coordinate system; $\alpha(x ; \beta)$ is the $x$-aligned wavenumber component of the crossflow mode of interest; and $\tilde{\phi}(x, y)$ represents an appropriately normalized local mode shape at station $x$. The cumulative coupling coefficient $\hat{C}(x)$ then denotes the effective amplitude of the crossflow mode at $x=x_{0}$; it is a function of both the intrinsic receptivity characteristics of the underlying boundary layer flow and the roughness geometry $F(x, z)$ :

$$
\begin{gathered}
\hat{C}(x)=\int_{x_{0}}^{x} \tilde{F}(\xi, \beta) \Lambda(\xi, \beta) \exp \left[-i \theta_{r}(\xi, \beta)\right] d \xi \\
\theta_{r}(x, \beta)=\int_{x_{0}}^{x} \alpha(\xi, \beta) d \xi
\end{gathered}
$$

where the lower limit of integration $x_{0}$ corresponds to the upstream end of the roughness distribution; $\tilde{F}(\xi, \beta)$ corresponds to the local amplitude of the relevant (spanwise) Fourier harmonic of the roughness height distribution $F(x, z)$, while $\Lambda(\xi, \beta)$ characterizes the efficiency of the local receptivity to surface roughness, i.e. the local Green's function with the response measured at the forcing location $\xi$. The combination of terms: $\Lambda(\xi, \beta) \exp [-i \theta(\xi, \beta)]$, is essentially an adjoint function that characterizes the impulse response, measured at the location $x$ to forcing at location $\xi$.

\section{Results}

\section{V.A. Incompressible Swept Hiemenz Flow}

The swept Hiemenz flow is an ideal configuration for concept study or code verification for crossflow instability in three-dimensional (3D) boundary layers. The basic flow has a linearly varying $x$ profile, given by selfsimilar boundary-layer equations, and a uniform spanwise velocity component in the free-stream. The predictions of localized receptivity for this flow were presented in Refs. ${ }^{15}$ and ${ }^{19}$ using non-parallel receptivity theory plus linearized Navier-Stokes, and a linearized PSE framework, respectively. It was shown that neglect of mean-flow non-parallelism led to considerable over-prediction of the efficiency function magnitude for receptivity due to short scale variations in surface geometry or surface suction velocity, but the results based on linearized PSE were in excellent agreement with those based on linearized Navier-Stokes equations. See references 15, 19,41, 42 for a more detailed discussion of swept Hiemenz flow.

The receptivity of the swept Hiemenz flow is considered first, beginning with a code-on-code (PSE/HLNS) validation exercise. The flow conditions are $R e=400$ and $\omega=0 ; \beta=0.4$, based on the following nondimensionalizations:

$$
\begin{gathered}
U_{\infty}^{*}=S^{*} X^{*} ; S^{*}=\partial U^{*} /\left.\partial X^{*}\right|_{x^{*}=0} ; l^{*}=\sqrt{\left(\nu^{*} / S\right)} \\
X=X^{*} / l^{*} ; R e=W_{\infty}^{*} l^{*} / \nu^{*} ; U_{\infty}=U_{\infty}^{*} / W_{\infty}^{*}=X / R e
\end{gathered}
$$

(Here, Re denotes the Reynolds number based on the spanwise velocity component along the attachment line and the length scale based on the velocity gradient in the chordwise direction.) Figure 1(a) compares the growth rate $\sigma_{u}$ of an $x$ velocity disturbance, as computed using the LASTRAC ${ }^{25}$ PSE and the present HLNS code. The growth rates are indistinguishable for this problem, verifying the parabolizing approximation in the PSE approach for problems of this type. Figure 1(b) compares the efficiency function associated with wall suction. The results agree well with those presented in references 15,19.

\section{V.A.1. The Adjoint Solution}

Figure 2(a) shows the Hiemenz flow $\Psi_{v}$-component of the adjoint solution for the HLNS equations. The cost functional is the magnitude of the velocity perturbation $\left(I_{U}\right)$ at $x=400$, a location sufficiently downstream for the flow perturbations to be completely dominated by the least stable crossflow instability excited upstream. Plotted as a function of the $x$ distance are the real and imaginary parts of the sensitivities as well as their magnitude envelope. Note that the data are plotted on a logarithmic-linear scale. The sensitivities of the $\mathrm{u}$ - and $\mathrm{w}$ - adjoint variables have a similar behavior. The most favorable location for a perturbation in the normal wall velocity is at approximately $x=160-170$ [i.e. close to the neutral location: $(x=162)$ for crossflow instability with $\beta=0.4$.] The streamwise wavelength of $\Psi_{v}$ at this location is 
approximately 7.85. Also note that the wavelength of $\Psi_{v}$ increases linearly with $x$, as does the velocity $U_{\infty}$ [see equation (15)].

Figure 2(b) shows the results of a grid refinement study for the Hiemenz flow, HLNS adjoint variable $\Psi_{v}$. Note that the data are plotted on a logarithmic-logarithmic scale. Four grids are used in this study, with $x$ resolution ranging from 1,920 - 5,120 collocation points. All grids were distributed linearly in the $x$ coordinate, to ensure a fixed number of points per wavelength. The normal resolution is held fixed at 55 Chebyshev collocation points in all cases. The data collapses when the adjoint variables are scaled by the local grid metric (in this case, the inverse streamwise grid spacing). which allows each grid to be compressed independently in the vicinity of roughness elements. Note the rapid convergence of the adjoint variable in the upstream portion of the flow, the region of most relevance to receptivity prediction. The grid convergence is slowest for locations between the location used to define the cost functional $(x=400)$ and the beginning of the buffer domain $(x=450)$, i.e. the region of least relevance to receptivity prediction. The resolution of the adjoint variable is far less demanding in the vicinity of the localized roughness elements, $x=160$, but requires greater resolution near the cost functional. In short, the grid resolution requirements needed to resolve the primal and adjoint variables can be very different.

Figure 2(b) also shows the results for a cost functional placed at $x=410$. The upstream results are indistinguishable (after appropriate scaling) from those obtained using $x=400$, while the solutions near the functional are quite different. Thus, locating the cost functional at a position far downstream from the neutral point (in flow of this kind) is sufficient to obtain a meaningful adjoint solution.

Surface roughness is an efficient mechanism for exciting crossflow disturbances if its spectral component at (or near) the disturbance wavelength is large. ${ }^{11,15,20,42}$ To this end, it is desirable to study the impact of roughness elements of various sizes on a fixed wavelength disturbance, or conversely, to study the impact of a fixed size roughness element on disturbances of various wavelengths. The adjoint HLNS solution procedure provides an extremely efficient means to accomplish these parametric studies; the first step of which is obtaining a high-quality adjoint solution for all the disturbance wavelengths in question. Figure 3 shows the HLNS primal and adjoint solutions for the swept Hiemenz flow for the conditions $R e=400, \omega=0$ for a decade variation in the spanwise parameter $\beta=0.1,0.2,0.4,0.6,0.8,1.0$. Figure $3(\mathrm{a})$ shows the influence of the parameter $\beta$ on disturbance growth and/or decay. In all cases, a suction hole is located on the surface at the spatial location $x=200$. Plotted on a linear-logarithmic scale is the variation in receptivity as a function of the forcing location, measured as a function of the magnitude of wall pressure fluctuation. The $\beta=0.4$ disturbance has the largest amplitude at the outflow plane while that of $\beta=1.0$ is the smallest. All disturbances decay super-exponentially ahead of the wall disturbance. Figure 3(b) shows the adjoint solution $\Psi_{v}$ as a function of the parameter $\beta$. Note that the adjoint solutions are qualitatively consistent with the growth rates shown in Fig. 3(a).

\section{V.A.2. Spanwise Periodic Roughness Arrays: DREs}

The definition of a spanwise periodic array of roughness elements has at least four independent parameters that can be optimized to accomplish a desired effect; 1 ) the roughness size and shape (e.g. cylindrical with radius $r$ ), 2) the roughness spacing (i.e. wavelength in spanwise direction), 3) the chordwise position on the airfoil, and 4) the height of the element. The first two are closely related (even dependent) and are used to change the relative mix of various harmonics in the boundary layer, while the third positions the disturbance at a desirable location (e.g. the neutral point in the stability curve). The fourth provides the amplitude of the disturbance. (Clearly the optimality of a particular DRE also depends on all the flow parameters $R e, \alpha_{A o A}, \cdots$ as well as the the spanwise mode $(\beta)$ in question.) We begin by optimizing the diameter/shape, spacing and position of a spanwise DRE array used to excite swept Hiemenz flow.

The shape of each element is assumed to be a circular cylinder, with the corners of the cylinder assumed to be sharp; rounding them had little impact. The radius of the circle was used as the first parameter in the study. The spanwise spacing for this exercise was chosen to coincide with the wavelength of the spanwise mode. Next, given a $\beta$ (and $\lambda=2 \pi / \beta$ ), an FFT was used at each $x$ position to expand the spanwise shape into its Fourier components, whereby the amplitude at the wavelength $\lambda$ was identified. Note that this amplitude varies with $x$ position, depending on the relative position on the element. For example, assume the radius to be half the spanwise period: $2 r=\lambda$. The spanwise shape in the middle of this element $x_{0}$ is a constant because the elements exactly touch in this case. All off-center spanwise shapes are square waves of shrinking breadth (with period $\lambda$ ), until the positions $x=x_{0} \pm r$ are reached, and are zero thereafter.

Figure 4 shows the effect of DRE radius and location on the amplitude of the crossflow mode corresponding 
to the fundamental harmonic of the array spacing. The flow conditions are $R e=400$ and $\omega=0$. Results for three spanwise wavenumbers $(\beta=0.2,0.40 .8)$ are shown in Figs. 4(a), 4(b), and 4(c), respectively. All results were obtained by convolving the adjoint solution for each respective $\beta$ with the DRE surface boundary condition. The adjoint cost functional used for this study was the surface pressure $I_{P}$, and was located sufficiently downstream to not influence the results. The abscissa in each plot is the element radius normalized by the spanwise wavelength $\lambda=2 \pi / \beta$ being considered. The ordinate is the amplitude of the surface pressure at the objective location. (Note the scale changes for each $\beta$ ). Five $x$ positions were tested for each spanwise $\beta$, centered approximately at the neutral point. Observations include

- The peak sensitivity coincides in all cases at the neutral point, with a modest decrease in sensitivity for upstream or downstream locations. This result follows immediately from the breadth of the adjoint solution [see Fig. 3(b)] in the vicinity of the neutral point.

- Cyclic peaks and attenuation's occur as the radius of the DRE is varied from $0 \leq r \leq \lambda$.

\section{V.A.3. Deterministic Nonlocal Roughness: Wavy Wall}

As a building block for distributed receptivity predictions, we extend the results in Ref. 19 to surface waviness of the form $F(x, z)=\exp \left[i\left(\alpha_{w} x+\beta z\right)\right]$ using both the linearized PSE and HLNS frameworks. (Only the HLNS results are presented herein, as the results obtained by both approaches are nearly indistinguishable; a further verification of the adjoint PSE predictions.) As discussed in Ref., ${ }^{43}$ predictions for such wavy wall configurations can be easily applied to estimate the receptivity due to a spatially homogeneous roughness distribution.

The $x$ evolution of the effective coupling coefficients for 13 values of $\alpha_{w}$ is shown in Fig. 5, calculated by convolving the Hiemenz wall adjoint solution with the individual surface waviness boundary condition. Each profile $\alpha_{w j}, j=1,13$ is plotted using the detuning parameter $d=\left(\alpha_{w}-\alpha_{\text {neutral }}\right) / \alpha_{\text {neutral }}$ where $\alpha_{\text {neutral }}$ is the local wavenumber at the lower branch neutral point. This definition of $d$ normalizes the individual difference in wavenumber $\alpha_{w}-\alpha_{\text {neutral }}$ against $\alpha_{\text {neutral }}$. Figures 5(a) and 5(b) display the spatial evolution of the effective coupling coefficient of the positively and negatively detuned disturbances, respectively, for the spanwise wavenumber $\beta=0.4$. Figures 5(c) displays the coupling coefficient at the spatial location $x=400$ as a function of the detuning parameter $d$, for four spanwise wavenumbers: $\beta=0.2,0.4,0.6,0.8$.

Figures 5(a) and 5(b) show that the receptivity to wavy walls is effectively confined to a relatively narrow region, centered on the location where the wavenumber $\alpha_{w}$ of the surface height distribution most closely matches the real part of the local instability wavenumber in the $x$ direction. Because chordwise wavenumber of the crossflow mode decreases with $x$, the wavenumber synchronization at smaller $\alpha_{w}$ occurs farther downstream, consistent with the progressively downstream movement of the region of rapid rise in $\hat{C}$ as the detuning parameter $d$ becomes more negative [see Fig. 5(b)]. The variation in the overall coupling coefficient (i.e., the value of the downstream asymptote of $\hat{C}$ ) with respect to $d$ is roughly symmetric about $d=0$, although the actual peak is slightly shifted to $d=-0.10$. This symmetric dependence on the detuning $d$ is similar to the case of acoustic excitation of Tollmien-Schlichting (TS) waves due to small amplitude waviness imposed on a flat surface. ${ }^{43}$ The striking difference between the two cases, however, corresponds to the range of the detuning parameter over which relatively significant receptivity occurs. Whereas the coupling coefficient becomes smaller than 10 percent of the peak value for $|d|>0.10$ for TS excitation over a flat plate, the swept Hiemenz flow appears to be receptive to surface waviness for detuning parameters in excess of $|d|>0.5$.

Figure 5(c) shows the overall effect of detuning at several spanwise wavenumbers $\beta$. Here, the downstream asymptote of the effective coupling coefficient is plotted as a function of the detuning parameter $d$. Unlike the $\beta=0.4$ case, detuning lacks symmetry for other values of $\beta$. Indeed, the decay in $\hat{C}$ for positive detuning is significantly slower than for negative detuning.

Table 1 compares the receptivity of local DREs and distributed wavy wall surface boundary conditions, for three values of the parameter $\beta$. The actual numbers in the table correspond to 1 ) the maximum response to local DREs of any size or surface location [i.e. the maximum amplitude derived from Figs. 4(a), 4(b), and $4(\mathrm{c})$ ] and 2) the maximum response to a distributed wavy wall for any value of detuning parameter $d$ [i.e. the maximum amplitude derived from Fig. 5(c)]. Recall that the adjoint cost functional used for both studies is the surface pressure $I_{P}$, which implies that this comparison relates the surface pressure at $x=400$ : a location far downstream from the region of peak sensitivity. Clearly, the distributed wavy wall is much more effective at exciting the growth of disturbances; over an order of magnitude in all cases. 


\begin{tabular}{||c|c|c|c||}
\hline \hline Surface & $\beta=0.2$ & $\beta=0.4$ & $\beta=0.6$ \\
\hline \hline DREs & $6 \mathrm{e}-6$ & $1 \mathrm{e}-4$ & $7 \mathrm{e}-6$ \\
\hline Wavy Wall & $1 \mathrm{e}-4$ & $4 \mathrm{e}-3$ & $3 \mathrm{e}-4$ \\
\hline
\end{tabular}

Table 1. Comparison of the amplitude of the pressure disturbance at $x=400$ achieved using DREs or wavy wall surface profiles .

\section{V.B. SWIFT Configuration}

A long term goal is to predict the initial crossflow amplitudes via a reduced order specification of the roughness geometry, using only a small number of amplitude and texture parameters for the surface roughness. It is envisioned that the study will help to identify the most relevant roughness specification parameters and hence, contribute to the potential development of an appropriate database pertaining to the natural roughness of typical aerodynamic surfaces. ${ }^{17}$ Here, we report the progress that has been made to date.

The study begins with a general description of the stability characteristics of the SWIFT configuration. Next, the adjoint solution is used to identify the highly sensitive regions near the nose, followed by a parametric study that identifies the optimal radius and placement of DREs. Finally, experimentally obtained surface roughness profiles are used to assess the receptivity characteristics. Receptivity predictions for the suction surface of the SWIFT configuration are presented. The flow conditions correspond to Test Point 27 , with $-4.69^{\circ}$ angle of attack and $R e_{c}=7.155 \times 10^{6}$. The selected spanwise station corresponds to an inboard section at 13 inches from the root (i.e., mounting) location of the test article.

Figure 6 shows the evolution of the crossflow disturbance amplitude in the chordwise direction $x$. The abscissa is the nondimensional distance from the stagnation streamline, while the ordinate is the amplitude of the energy

$$
E(x)=1 / 2 \int_{0}^{y \max }\left[u(y, x) u^{*}(y, x)+v(y, x) v^{*}(y, x)+w(y, x) w^{*}(y, x)\right] d y
$$

associated with a crossflow mode with a spanwise wavelength of $4.5 \mathrm{~mm}$. Figure $6(\mathrm{a})$ shows the disturbance growth on the scale of the chord, while Fig. 6(b) shows a magnified view of the nose region. Each plot compares the solutions obtained with the HLNS solver on grids of different density, and that obtained from the linear PSE solver LASTRAC. ${ }^{19}$ Excellent agreement is achieved throughout most of the domain, until the buffer domain region $(0.55 \leq x)$ is encountered in the HLNS formulation. The PSE calculation begins near $x \approx 15 \mathrm{~mm}$, close to the neutral point of the crossflow mode. The HLNS solution corresponds to the forced response of the boundary layer to a spanwise periodic array of DREs near $x \approx 10 \mathrm{~mm}$. Because of the linearity of both solutions, the PSE solution is scaled to match the HLNS solution at $x=300 \mathrm{~mm}$.

\section{V.B.1. The Adjoint Solution}

Figure 7(a)-(b) shows the solutions to the adjoint HLNS equations for the surface variables $\Psi_{u}, \Psi_{v}, \Psi_{w}$. The objective function used for these solutions was the surface pressure $I_{P}$ at the point $x=0.5$ chord. The real and imaginary components and the magnitude of each surface variable, e.g. $\left[\operatorname{Re}\left(\Psi_{u}\right)\right],\left[\operatorname{Im}\left(\Psi_{u}\right)\right]$, and $\left|\Psi_{u}\right|$, are plotted. Figure 7(b) is a coarser view of Fig. 7(a) used to compare the sensitivities $\Psi_{u}$ and $\Psi_{w}$ to forcing via the $u$ and $w$ wall components, respectively. The most sensitive portion on the surface of the airfoil, is located upstream of the $x=2 \%$ chord (i.e. $x \leq 27 \mathrm{~mm}$ ) and then decreases rapidly with distance downstream of this peak location. The significance of receptivity within the leading edge region is consistent with both previous experiments ${ }^{22}$ and theoretical predictions. ${ }^{12,16,17,19}$ Also note that the chordwise wavelength of the adjoint components decreases rapidly in this region.

Figure 8 extends the results shown in Fig. 7 to include the influence of different spanwise wavelengths. Shown are the amplitudes of the adjoint variable $\Psi_{v}$ over the first $100 \mathrm{~mm}$ of the chordwise distance. Spanwise wavelengths in the range $2.25 \mathrm{~mm} \leq \lambda \leq 21.5 \mathrm{~mm}$ are included in the study. Figure 8 (a) shows the amplitude of the adjoint variable $\left|\Psi_{v}\right|$ for all wavelengths, as well as $R e\left[\Psi_{v}\right]$ for the $\lambda=2.25 \mathrm{~mm}$ and $\lambda=21.5 \mathrm{~mm}$ cases. The wave nature of the chordwise wall sensitivity is clearly evident in the $\operatorname{Re}\left[\Psi_{v}\right]$ profiles shown for $\lambda=2.25 \mathrm{~mm}$ and $\lambda=21.5 \mathrm{~mm}$. Note that the chordwise wavelength of the sensitivity, scales with the spanwise wavelength $\lambda$ near the nose and expands rapidly beyond approximately $x=10 \mathrm{~mm}$. 


\section{V.B.2. Spanwise Periodic Roughness Arrays: DREs}

Figure 9 shows convolutions of the adjoint variables shown in Fig. 7 against surface boundary conditions approximating DREs. The parameter investigation is equivalent to that performed for the swept Hiemenz flow (see Fig. 4); specifically, the shape of each DRE is assumed to be a circular disk of variable radius $r$, with spanwise spacing $\lambda=2 \pi / \beta$. The position of the DRE array is varied over the interval $0 \leq x \leq 8 \lambda$, which spans the neutral point on the surface of the airfoil and most of the highly receptive region near the nose. The abscissa (linear) of Fig. 9 shows the radius of the DRE normalized by the spanwise wavelength $\lambda$, while the ordinate (linear) shows the amplitude of the wall pressure at the mid-chord location. (All calculations were performed using the adjoint HLNS formulation, with the $I_{P}$ cost functional situated mid-chord.)

The spanwise oriented DRE array is highly effective at exciting the crossflow disturbance within the range $x \approx(3-4) \lambda$ which is $13.5-18 \mathrm{~mm}$ from the attachment line. Note that this location is close to the neutral point identified by the PSE computation shown in Fig. 6(b). The amplitude in this region [e.g. $x=(2.0-4.0) \lambda]$ is relatively uniform, but decreases rapidly outside the interval. The receptivity falls to near zero as the radius of the DRE approaches $2 r=\lambda$.

In general, the DRE results for the SWIFT airfoil are similar to those obtained for DREs subjected to the swept Hiemenz mean flow. Specifically, the peak amplitude coincides with the peak of the adjoint, and there is slow decay in peak amplitude for upstream or downstream locations. Subtractive cancellation occurs as the radius of the DRE traverses through resonant wavelengths. The region $13.5-40 \mathrm{~mm}$ seems a likely candidate for a wavy wall surface study, and is the object of ongoing work.

\section{V.B.3. Random Surface Roughness: Experimental Data}

The final exercise is to establish the disturbance behavior resulting from physically realistic surfaces. Several different surface textures/finishes were applied to the SWIFT airfoil to study the impact of these textures on transition. The data were provided by Saric, ${ }^{44}$ taken from an ongoing Texas A\&M University roughness/transition experiment. The surface textures included regions with the following distinguishing features: 1) a taped surface, 2) a polished surface, 3) a painted surface, and 4) a painted surface that was subsequently sanded. The physical characteristics of each texture were obtained experimentally using a stylus that sweeps linearly along the spanwise direction. The sweep length is 1.6 inches over which 5,000 datapoints were collected. In total, 13 distinct surface samples were collected from four texture groups.

Table 2 presents the data used to quantify the 13 surface samples, and as expected each texture has its own characteristics. Cases $1-4,5-9,10-11$ and $12-13$ were obtained from the 1) taped, 2) polished, 3) painted and 4) painted/sanded categories, respectively. The metrics used to characterize the surface textures are described in the caption of the figure, e.g. $\left\|h_{\text {raw }}\right\|_{1}=$ raw mean surface absolute deviations. Several observations about the surface data are worthy of note.

- The painted surface has by far the roughest texture, while the polished surface is the smoothest. They differ in RMS surface deviation by over an order of magnitude.

- There is considerable deviation within the raw data for each individual texture group. For example, the raw data reported in case 07 is significantly different from the other members of the polished group.

- A spectral decomposition is needed to quantify the amplitude of each wavenumber component of the surface height distribution. Because the data are neither periodic nor smooth, this decomposition is problematic if Fourier techniques are used. Thus, a detrending step (described shortly) or equivalently a high pass filter, is necessary to correct for the large scale variations comparable to the length of each scan.

- Detrending the data profoundly changes the values of some of the metrics used to characterize the surface texture. Apostori testing must be used to establish the efficacy of the detrending step.

- Changes in raw maximum distance peak to peak: $\zeta_{3}$ and raw five point average maximum distance peak to peak: $\zeta_{4}$, as a result of detrending were not checked.

Figure 10(a) shows an example of how the surface data are detrended. The data being detrended is reported in Table 2 as Case 3, and belongs to the taped group. In Fig. 10(a), the raw data (shown in red) has significant long wavelength variation. Superimposed along this long wavelength profile are high 


\begin{tabular}{||c||c|c|c|c|c||c|c||}
\hline \hline \multicolumn{1}{||c||}{} & \multicolumn{5}{c||}{ Raw Data } & \multicolumn{2}{c||}{ Detrended } \\
\hline \hline \multirow{3}{*}{ Taped } & Case & $\left\|h_{\text {raw }}\right\|_{1}$ & $\left\|h_{\text {raw }}\right\|_{2}$ & $\zeta_{3}$ & $\zeta_{4}$ & $\left\|h_{\text {det }}\right\|_{1}$ & $\left\|h_{\text {det }}\right\|_{2}$ \\
\hline \hline \multirow{5}{*}{ Polished } & 01 & 0.84 & 0.99 & 3.97 & 3.66 & 0.06 & 0.08 \\
& 02 & 1.32 & 1.55 & 5.49 & 5.36 & 0.09 & 0.11 \\
& 03 & 1.29 & 1.52 & 5.44 & 5.32 & 0.08 & 0.11 \\
& 04 & 0.82 & 1.00 & 4.69 & 3.85 & 0.09 & 0.12 \\
\hline \multirow{3}{*}{ Painted } & 05 & 0.12 & 0.17 & 1.53 & 0.92 & 0.08 & 0.11 \\
& 06 & 0.12 & 0.15 & 1.14 & 0.86 & 0.06 & 0.08 \\
& 07 & 0.31 & 0.36 & 1.89 & 1.18 & 0.09 & 0.11 \\
& 08 & 0.15 & 0.17 & 1.10 & 0.84 & 0.07 & 0.10 \\
& 09 & 0.15 & 0.18 & 1.19 & 0.74 & 0.07 & 0.08 \\
\hline \multirow{2}{*}{ Painted/Sanded } & 10 & 6.27 & 8.06 & 48.12 & 42.22 & 6.06 & 7.86 \\
& 11 & 4.37 & 6.00 & 49.16 & 30.16 & 4.35 & 5.93 \\
& 12 & 1.08 & 1.18 & 16.25 & 13.49 & 0.58 & 1.08 \\
& 13 & 0.95 & 1.78 & 6.14 & 3.29 & 0.50 & 0.95 \\
\hline \hline
\end{tabular}

Table 2. SWIFT experimental data: surface roughness statistics. The column designations are as follows; $\left\|h_{\text {raw }}\right\|_{1}=$ Raw mean of surface absolute deviations; $\left\|h_{\text {raw }}\right\|_{2}=$ Raw root mean square of surface deviations; $\zeta_{3}=$ Raw maximum distance peak to peak; $\zeta_{4}=$ Raw five point average maximum distance peak to peak; $\left\|h_{d e t}\right\|_{1}=$ Detrended mean of surface absolute deviations; $\left\|h_{d e t}\right\|_{2}=$ Detrended root mean square of surface deviations.

frequency surface oscillations which appear to be random. Detrending the data begins by first projecting the raw data onto a low-order polynomial using an $L_{2}$ minimization of the deviation. This allows the coefficients of a long wavelength polynomial (shown in green), to be determined. Subtracting the low-order polynomial from the original data produces a detrended dataset (shown in blue), that is approximately periodic. The polynomial order used to detrend all data in this study was 4; polynomials of degree higher than 4 decrease the energy of the modes of most interest, while those of lower degree were inadequate for the purpose of detrending.

Figure 10(b) compares the Fourier spectrum of the original raw data (solid lines), with that of the detrended data (dashed lines). The four cases shown all originate from the polished surface group. Note that detrending the original data is highly effective at reducing the first three Fourier modes $(\lambda \geq 15 \mathrm{~mm})$, while leaving the high frequency character of all other modes. Furthermore, note that the Fourier modes corresponding to the most unstable mode of the crossflow instability $(4.5 \mathrm{~mm})$ remain virtually unaffected by the detrending step.

Figures 11 and 12 show the original raw and detrended data for the polished cases and the painted and painted/sanded cases. Note that detrending the painted data has little effect on the apparent shape of the surface profiles. This is consistent with the data in Table 2 which indicates that the raw surface measures: $\left\|h_{\text {raw }}\right\|_{1},\left\|h_{\text {raw }}\right\|_{2}$, closely match the detrended measures: $\left\|h_{\text {det }}\right\|_{1},\left\|h_{\text {det }}\right\|_{2}$.

\section{V.B.4. Random Surface Roughness: Disturbance Growth}

To determine the impact of the different textures on crossflow disturbance amplitude, the near leading edge surface of the SWIFT airfoil is fitted with each of the 13 experimentally measured roughness patches. The region $0 \leq x \leq 0.02 \%$ chord $(0 \leq x \leq 27 \mathrm{~mm})$ was shown previously (see Figs. 7 and 9 herein), to be the most efficient at exciting the crossflow disturbances. The patches extend approximately $40 \mathrm{~mm}$ chordwise, allowing each to completely cover the sensitive nose region.

An assumption is necessary in the chordwise direction to fully specify the surface profile. For brevity, roughness characteristics along the chordwise direction are assumed to be the same as those in the spanwise direction, and that the airfoil surface is composed of a tensor-product roughness patch for each of the 13 surface roughness patches.

Using the surface roughness patches as boundary conditions, the adjoint HLNS methodology is then used to predict the crossflow disturbance amplitude for each texture. Convolving each surface boundary condition 
with the adjoint solution, $\Psi_{\beta}$, yields the crossflow disturbance amplitude at the location of the specified cost functional. The $I_{U}$ cost functional is used exclusively in this study and is always located at the mid-chord position.

Figures 13 and 14 present an extensive study of disturbance sensitivity to surface roughness textures. The abscissa (linear) of each figure covers the spanwise wavenumber interval $100 \leq \beta \leq 5000$, (i.e. $86 \mathrm{~mm} \geq \lambda^{*} \geq 1.7 \mathrm{~mm}$; lengths nondimensionalized by 4.5' chord) approximately centered about the highly unstable $\beta=1915$ wavenumber (i.e. the $4.5 \mathrm{~mm}$ spanwise wavelength). The ordinate (logarithmic) measures the disturbance amplitude for each test case.

Each of the two figures contains 611 symbols; 13 symbols (each corresponding to an individual surface texture patch) at 47 distinct wavenumbers $\beta$. The symbols for the 13 surface texture patches form a vertical distribution at each wavenumber $\beta$. Recall that the 13 surface textures were divided into four groups: taped, polished, painted, and painted/sanded. Distinct symbols (and colors) are used for each surface texture group. For example, the green inverted triangles denote the disturbance amplitude resulting from the two painted surface patches at each of the 47 wavenumbers.

Each of the 611 symbols from Figs. 13 and 14 is accompanied by an overbar ${ }^{-}$. The over-bars quantify the 1) ensemble mean disturbance amplitude (with 128 realizations) and 2) $R M S$ deviation in response to one surface roughness patch. (The bottom half of the $R M S$ deviation bar is omitted for reasons of clarity.) The ensemble of roughness distributions is constructed as follows. First, note that the phase of the data in each roughness patch is completely arbitrary because of periodicity (an assumption shown to be essentially valid in the detrended case). Thus, an arbitrary number of the disturbance responses can be obtained from the data in a single roughness patch. For example, assume a roughness patch extends over the surface interval $0 \leq x \leq 40 \mathrm{~mm}$, but that the data are translated periodically $N$ times, such that the virtual origin $x_{0}$ (the first data point) satisfies the relation $x_{0}=(k-1) 40 \mathrm{~mm} / N, k=1, N$. Indeed, the shift could even be random. Using this periodic translation of boundary data, an ensemble mean and standard deviation in disturbance amplitude can be determined for each surface patch. Numerical experimentation determined that the mean and RMS are converged to three significant digits using an ensemble of 128 samples. ${ }^{\mathrm{c}}$

Figures 13 and 14 are obtained using the raw and detrended data, respectively. Note that there is little difference in disturbance amplitudes for wavenumbers in the interval $\beta \geq 1300(\approx 6.5 \mathrm{~mm})$ and no difference for $\beta \geq 1915(\approx 4.5 \mathrm{~mm})$. The differences in receptivity predictions without and with detrending of surface height distributions, are consistent with the associated variation in the Fourier spectra of the surface height distribution. Specifically, the effect of detrending is to decrease the amplitudes of the long wavelength modes (e.g. $\lambda \geq 15 \mathrm{~mm}$ ), which are outside the wavelength band that is relevant for transition as well as transition control via DREs.

The following observations summarize this study on the effects of random surface roughness on the strength of crossflow disturbance excitation.

- The most striking result is the wide range of crossflow amplitudes resulting from the four surface textures. Approximately two orders of magnitude variation in disturbance amplitude is observed when comparing the painted and polished surfaces. The differences in predicted disturbance amplitudes for the painted/sanded leading edge and the polished edge are smaller, but still approximately one order of magnitude.

- In addition to the surface metrics, $\left\|h_{\text {raw }}\right\|_{k}, k=1,2 ; \zeta_{k}, k=3,4$ and $\left\|h_{\text {det }}\right\|_{k}, k=1,2$, an accurate Fourier decomposition of the surface roughness is needed to predict the disturbance response at all wavenumbers. Non-periodicity and aliasing can lead to erroneous component amplitudes.

- The range of mean disturbance amplitudes ( $\max$ - min) arising from the individual members of a texture group, greatly exceeds the RMS variation in the individual ensemble averages within that group. This effect could be greatly mitigated by surface samples of significantly greater length. Furthermore, the in-group deviation depends strongly on the disturbance wavenumber.

The effect of surface quality on transition has been documented in Ref. 1, wherein transition was noted to move from somewhere between 25 to 30 percent chord to 80 percent chord when the painted leading edge was polished to reduce the roughness amplitude from $1.0 \mu \mathrm{m}$ RMS (3.8 $\mu \mathrm{m}$ average peak-to-peak) to $0.3 \mu \mathrm{m}$ RMS (2.2 $\mu \mathrm{m}$ average peak-to-peak). Had linear stability based N-factor methods been applied to

${ }^{\mathrm{c}}$ Figures 13 and 14 are each the result of $128 \times 13 \times 47=78,208$ individual convolutions of surface data with the 47 adjoint solutions. The adjoint HLNS capability is enabling for problems of this complexity. 
predict the onset of transition in this case, the correlating $\mathrm{N}$-factor would have increased from 8 to 16 . The substantially smaller initial crossflow amplitudes on the polished leading edge (Fig. 14) are qualitatively consistent with the large delay in measured transition location.

Transition prediction via N-factor correlations for high-frequency secondary instabilities of the stationary crossflow modes was considered in Ref. 24 in the context of modeling transition delay via discrete roughness elements (DREs). By applying the same methodology in the absence of any DREs, transition onset location was predicted to shift downstream by approximately 15 percent of the chord length for each orderof-magnitude reduction in the initial amplitude of the dominant $4.5 \mathrm{~mm}$ stationary crossflow mode. The predicted shift in transition location could be considerably larger if only one of the two dominant types of secondary instability modes (namely, the family of secondary instability modes that is associated with spanwise gradients of the basic state, as against wall-normal gradients) were to be realized in practice. Because the predicted impact of the reduced crossflow receptivity on the polished leading edge appears to be smaller than that measured during the SWIFT experiments, further work is necessary to fully explain the observed transition behavior.

\section{Summary}

This paper extends the prediction capability for roughness induced crossflow receptivity to realistic surface roughness distributions, to pave the way for integrated, high fidelity analysis methods for crossflow transition, in general, and DRE based laminar flow control, in particular. An illustrative set of results relevant to a unique experiment involving a swept wing configuration were presented above.

The monumental scope of the problems at hand (e.g. quantification of flow response to random surface roughness via Monte-Carlo simulation of canonical and realistic geometries) made it expedient to develop an adjoint HLNS capability, to complement an existing HLNS solver. A discrete adjoint approach was adopted because of complexities resulting from the artificial boundary conditions used at the outflow plane of the HLNS solve and the fact that the discrete adjoint is an exact adjoint to the forward problem in question. The new adjoint formulation was exercised on a set of well known crossflow stability problems including the swept Hiemenz flow.

The new adjoint formulation was used to tackle the problem of distributed receptivity: excitation of stationary crossflow modes via a wavy wall $F(x, z)=\exp \left[i\left(\alpha_{w} x+\beta z\right)\right]$ was investigated in the context of swept Hiemenz flow. The receptivity to wavy walls is effectively confined to a relatively narrow region centered on the location where the wavenumber $\alpha_{w}$ of the surface height distribution most closely matches the real part of the local instability wavenumber along the chordwise $x$ direction. The variation in the overall coupling coefficient with respect to a detuning parameter $d=\left(\alpha_{w}-\alpha_{\text {neutral }}\right) / \alpha_{\text {neutral }}$, is roughly symmetric about $d=0$ (although the actual peak is slightly shifted to $d=-0.10$ ), similar to the case of acoustic excitation of TS waves due to waviness over a flat surface. ${ }^{43}$ However, the striking difference between the two cases corresponds to the range of detuning parameter over which relatively significant receptivity occurs. Whereas the coupling coefficient becomes smaller than 10 percent of the peak value for $|d|>0.10$ for TS excitation over a flat plate, the swept Hiemenz flow appears to be receptive to surface waviness for detuning parameters in excess of $|d|>0.5$.

Finally, the adjoint formulation was used to study the SWIFT experiment. One long term goal of this study is to enable an estimation of initial crossflow amplitudes via a reduced order specification of the roughness geometry, in the form of a small number of amplitude and texture parameters for the surface roughness. The study began with a general description of the stability characteristics of the SWIFT configuration, and confirmed that the most sensitive region is near the neutral point. DREs located in this region have the most impact. Next, the adjoint solution was used study the impact of different surface roughness patches on disturbance growth of unstable crossflow modes. An exhaustive exercise for 47 distinct spanwise wavelengths resulted in the following observations: 1) two orders of magnitude variation in disturbance amplitude are observed when comparing the different surface textures (painted vs. polished surfaces). This is qualitatively consistent with the significant shift in predicted transition location when the initial crossflow amplitude is reduced by one to two orders of magnitude. 2) Accurate Fourier decomposition of the surface roughness is needed to predict the disturbance response at all wavenumbers. 3) Significant variation in disturbance amplitudes appears within each of the four texture groups, and is greater than the RMS deviation predicted within each of the 13 individual surface samples. Much work still remains to establish a reliable reduced set of surface metrics. 
Much work is still left to be done regarding the quantification of surface roughness. The results presented herein are encouraging in suggesting that a low dimensional surface parametrization could eventually be identified. Ultimately, modeling random, spatially distributed small magnitude roughness on this and other canonical configurations will aid in the development of improved metrics (or improved interpretation of existing set of metrics) for the specification of natural surface roughness (e.g., RMS, peak-to-peak, spectral probability density function model); the end result being a more reliable set of assessment/design tools for DRE configurations and fast estimates of initial crossflow amplitudes for nonlinear PSE calculations with or without DREs.

\section{Acknowledgments}

The authors are thankful to Prof. William Saric of Texas A\&M University for providing the geometry, surface pressure distributions, and roughness measurements for the SWIFT flight configuration. The work described in this paper has been carried out under the Subsonic Fixed Wing project of NASA's Fundamental Aeronautics Program, as part of a partnership effort between NASA, AFRL and Texas A\&M University.

\section{References}

\footnotetext{
${ }^{1}$ Carpenter, A., Saric, W., and Reed, H., "Laminar Flow Control on a Swept Wing with Distributed Roughness," AIAA Paper 2008-7335, 2008.

${ }^{2}$ Arnal, D., Private communication, 2006.

${ }^{3}$ Saric, W. S., Carillo, R. B., and Reibert, M. S., "Leading Edge Roughness as a Transition Control Mechanism," AIAA Paper 98-0781, Jan. 1998.

${ }^{4} \mathrm{Li}, \mathrm{F}$. and Choudhari, M., "Spatially Developing Secondary Instabilities and Attachment Line Instability in Supersonic Boundary Layers," AIAA Paper 2008-590, 2008.

${ }^{5}$ Choudhari, M. and Streett, C. L., "Boundary Layer Receptivity Phenomena in Three-Dimensional and High-Speed Boundary Layers," AIAA Paper 1990-5258, 1990.

${ }^{6}$ Goldstein, M. E., "Scattering of Acoustic Waves into Tollmien-Schlichting Waves by Small Streamwise Variations in Surface Geometry," J. Fluid Mech., Vol. 154, 1985, pp. 509-529.

${ }^{7}$ Ruban, A. I., "On the Generation of Tollmien-Schlichting Waves by Sound," Fluid Dyn., Vol. 19, 1985, pp. $709-716$.

${ }^{8}$ Collis, S. and Dobrinsky, A., "Evaluation of Adjoint Based Methods for the Prediction of Linear and Nonlinear Receptivity," Proc. of the 5th IUTAM Symposium on Laminar-Turbulent Transition, 1999.

${ }^{9}$ Piot, E., Content, C., and Casalis, G., "Receptivity of Crossflow Instabilities to a Periodic Roughness Array on a Swept Cylinder: Investigation of the Roughness Size Influence," AIAA Paper 2008-502, 2008.

${ }^{10}$ Choudhari, M. and Streett, C. L., "Theoretical Prediction of Boundary-Layer Receptivity," AIAA Paper 1994-2223, 1994.

${ }^{11}$ Crouch, J. D., "Receptivity of Boundary Layers," AIAA Paper 1994-2224, 1994.

${ }^{12}$ Choudhari, M., Li, F., Chang, C.-L., and Malik, M., "NASA/Boeing HLFC Experiment: Data Analysis I," High Technology Corporation Report 1996-07, 1996.

${ }^{13}$ Streett, C. L., "Direct Harmonic Navier-Stokes Methods for Efficient Simulation of Weakly-Nonlinear Wave Packets," AIAA Paper 1998-0784, 1998.

${ }^{14} \mathrm{Ng}$, L. and Crouch, J., "Roughness-induced Receptivity to Crossflow Vortices on a Swept Wing," Phys. Fluids, Vol. 11, 1999, pp. 432-438.

${ }^{15}$ Bertolotti, F. P., "Receptivity of Three-Dimensional Boundary-Layers to Localized Wall Roughness and Suction," Phys. Fluids, Vol. 12, No. 7, 2000, pp. 1799-1809.

${ }^{16}$ Janke, E., "Receptivity and Transition Control of Swept-Wing Boundary-Layers; Effects of Surface Curvature and Nonlinearity," AIAA Paper 2001-2980, 2001.

${ }^{17}$ Choudhari, M., Chang, C.-L., Streett, C. L., and Balakumar, P., "Integrated Transition Prediction: A Case Study in Supersonic Laminar Flow Control," AIAA Paper 2003-0973, 2003.

${ }^{18}$ Jiang, L., Choudhari, M., Chang, C.-L., and Liu, C., "Direct Numerical Simulations of Crossflow Disturbances in Supersonic Boundary Layers," AIAA Paper 2004-589, 2004.

${ }^{19}$ Chang, C.-L. and Choudhari, M., "Boundary-Layer Receptivity and Integrated Transition Prediction," AIAA Paper 2005-0526, 2005.

${ }^{20}$ Choudhari, M., "Roughness-Induced Generation of Crossflow Vortices in Three-Dimensional Boundary Layers," Theor. and Comp. Fluid Dyn., Vol. 5, Feb. 1994, pp. 1-31.

${ }^{21}$ Radeztsky, R., Reibert, M., and Saric, W., "Effect of Isolated Micron-Sized Roughness on Transition in Swept-Wing Flows," AIAA J., Vol. 37, No. 11, pp. 1370-1377.

${ }^{22}$ Rhodes, R., Carpenter, A., Reed, H., and Saric, W., "CFD Analysis of Flight-Test Configuration for LFC on Swept Wings," AIAA Paper 2008-7336, 2008.

${ }^{23}$ Reibert, M., Saric, W., Carrillo, R. J., and Chapman, K., "Experiments in nonlinear saturation of stationary crossflow vortices in a swept-wing boundary layer," AIAA Paper 1996-0184, 1996.

${ }^{24}$ Li, F., Choudhari, M., Chang, C.-L., Streett, C., and Carpenter, M., "roughness Based Crossflow Transition Control: A Computational Assessment," AIAA Paper 2009-4105, 2009.
} 
${ }^{25}$ Chang, C.-L., "Langley Stability and Transition Analysis Code (LASTRAC) Version 1.2 User Manual," 2004, pp. 1-100. ${ }^{26}$ Streett, C. and Macaraeg, M., "Spectral Multidomain for Large-Scale Fluid Dynamic Simulations," Int. J. Appl. Numer. Math., Vol. 6, 1989.

${ }^{27}$ Pironneau, O., "On Optimum Profiles in Stokes Flow," J. Fluid Mech., Vol. 59, 1973, pp. 117-128.

${ }^{28}$ Pironneau, O., "On Optimum Design in Fluid Mechanics," J. Fluid Mech., Vol. 64, 1974, pp. 97-110.

${ }^{29}$ Pironneau, O., "Optimal Shape Design for Elliptic Systems," 1982.

${ }^{30}$ Jameson, A., "Aerodynamic Design Via Control Theory," J. Scientific Computing, Vol. 3, 1988, pp. 237-260.

${ }^{31}$ Jameson, A., Optimum Aerodynamic Design Using Control Theory, 1995, pp. 495-528.

32 Jameson, A., Pierce, N., and Martinelli, L., "Optimum Aerodynamic Design Using the Navier-Stokes Equations," J. Theor. Comput. Fluid Mech., Vol. 10, 1998, pp. 213-237.

${ }^{33}$ Anderson, W. K. and Venkatakrishnan, V., "Aerodynamic Design Optimization on Unstructured Grids with a Continuous Adjoint Formulation," AIAA Paper 97-0643, 1997.

${ }^{34}$ Anderson, W. K. and Bonhaus, D. L., "Airfoil Design on Unstructured Grids for Turbulent Flows," AIAA J., Vol. 37, No. 2, 1999, pp. 185-191.

${ }^{35}$ Nielsen, E. and Anderson, W. K., "Aerodynamic Design Optimization on Unstructured Meshes Using the Navier-Stokes Equations," AIAA J., Vol. 37, No. 11, 1999, pp. 957-964.

${ }^{36}$ Giles, M. and Pierce, N., "An introduction to the adjoint approach to design," Flow Turb. Comb., Vol. 65, No. 3-4, 2000, pp. 393-415.

${ }^{37}$ Giles, M., Duta, M., Muller, J., and Pierce, N., "Algorithm developments for discrete adjoint methods," AIAA J., Vol. 41, No. 2, 2003, pp. 198-205.

${ }^{38}$ Park, M., "Adjoint-based, three-dimensional error prediction and grid adaptation," AIAA J., Vol. 42, No. 9, 2004, pp. $1854-1862$.

${ }^{39}$ Nielsen, E. and Park, M. A., "Using an Adjoint Approach to Eliminate Mesh Sensitivities in Computational Design," AIAA J., Vol. 44, No. 5, 2006, pp. 948-953.

${ }^{40}$ Giles, M. and Pierce, N., "Adjoint equations in CFD: duality, boundary conditions and solution behaviour," AIAA Paper 97-1850, 1997.

${ }^{41}$ Malik, M., Li, F., and Chang, C.-L., "Cross-Flow Disturbances In 3-Dimensional Boundary-Layers - Nonlinear Development, Wave Interaction and Secondary Instability," J. Fluid Mech., Vol. 268, 1994, pp. 1-36.

${ }^{42}$ Schrader, L., Brandt, L., and Henningson, D., "Receptivity Mechanisms in Three-Dimensional Boundary-Layer Flows," J. Fluid Mech., Vol. 618, 2009, pp. 209-241.

${ }^{43}$ Choudhari, M., "Boundary-Layer Receptivity due to Distributed Surface Imperfections of a Deterministic or Random Nature," Theor. and Comp. Fluid Dyn., Vol. 4, No. 3, Feb. 1993, pp. 101-118.

${ }^{44}$ Saric, W., "Surface profile data from an ongoing troughness/transition experiment," Private Communication.

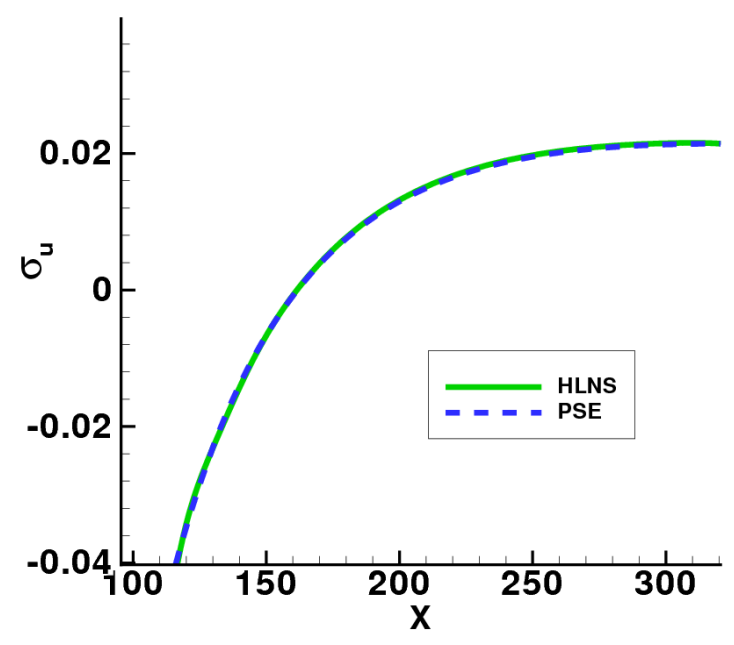

(a) Streamwise velocity growth rate.

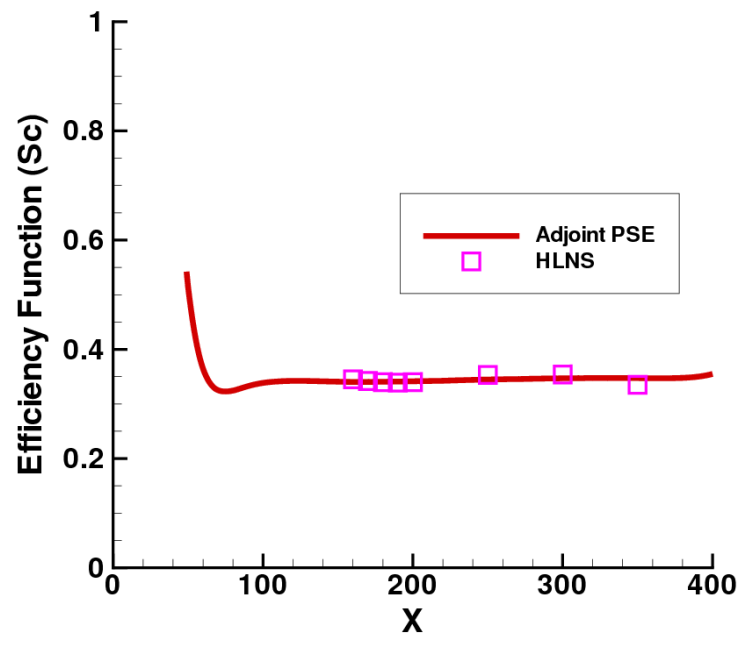

(b) Efficiency Function.

Figure 1. Comparison of the PSE and HLNS formulations on swept Hiemenz flow, Re $=400, \beta=0.4$ with suction surface boundary conditions. 


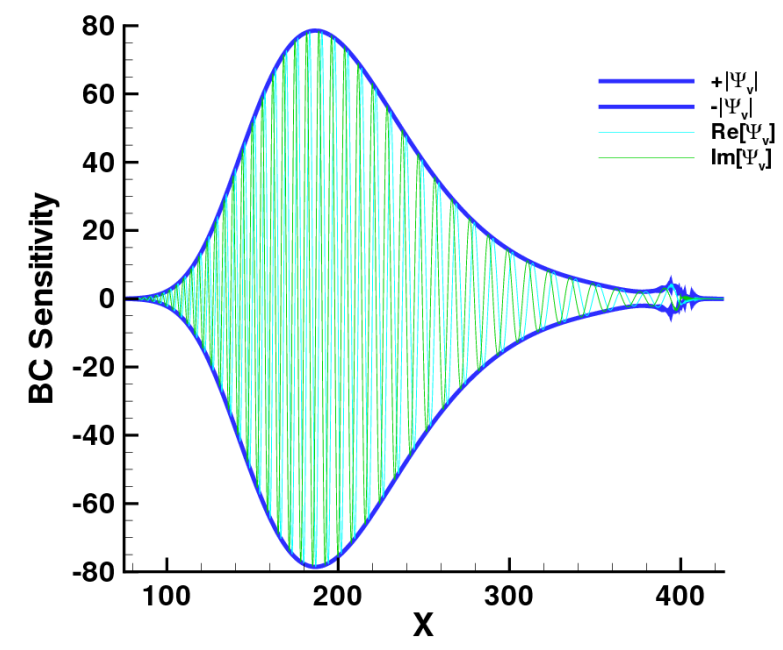

(a) Wall sensitivity to normal perturbations.

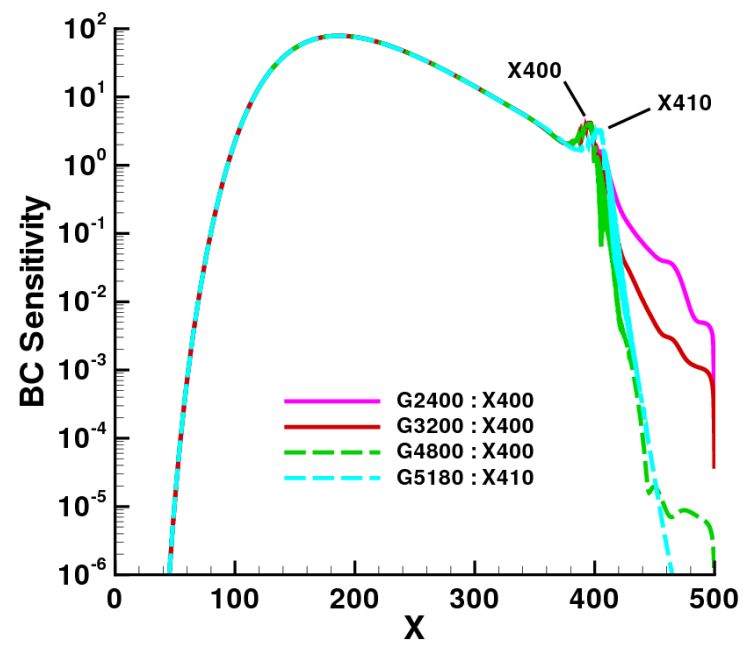

(b) Grid convergence of discrete adjoint.

Figure 2. Adjoint solution for the swept Hiemenz flow for conditions $R e=400, \beta=0.4$. Figure (a) shows the surface sensitivities of the real, imaginary and magnitude of $\Psi_{v}$; the cost functional $I_{U}$ is situated at $x=400$. Figure (b) shows a grid refinement study of the adjoint surface variable $\Psi_{v}$, as well as the influence of the location of $I_{U}$ on $\Psi_{v}$.

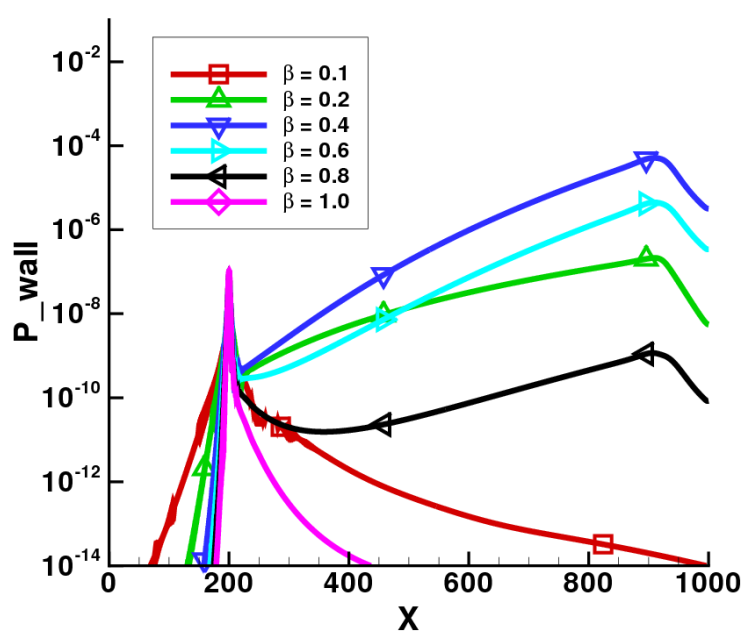

(a) Primal HLNS: Wall pressure for various spanwise $\beta$.

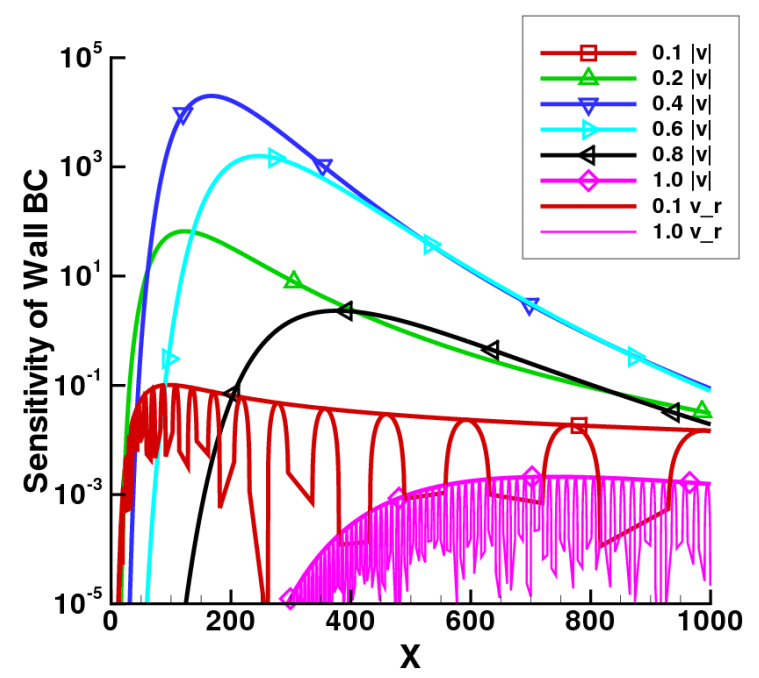

(b) Adjoint HLNS: $\Psi_{v^{-}}$sensitivity for various $\beta$.

Figure 3. HLNS primal and adjoint solutions for the swept Hiemenz flow: $(R e=400, \omega=0)$, comparing the influence of $\beta$ on disturbance growth. Figure (a) shows the $\beta$ sensitivity on growth and/or decay of disturbances initiated by wall suction located at $x=200$. Note that the buffer domain begins at $x=950$. Figure (b) shows the adjoint solution $\Psi_{v}$ as a function of the same $\beta$ parameters. 


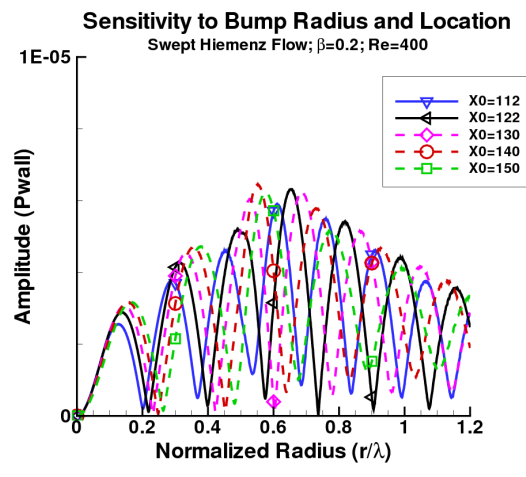

(a) HLNS: $\beta=0.2$.

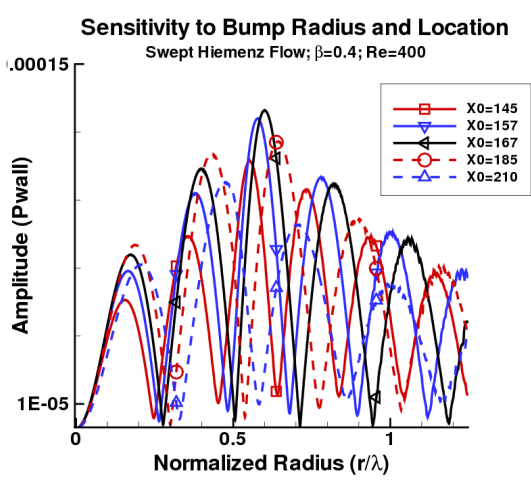

(b) HLNS: $\beta=0.4$.

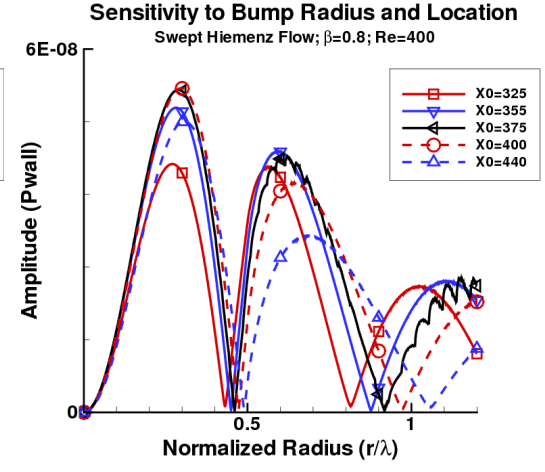

(c) HLNS: $\beta=0.8$.

Figure 4. HLNS: Radius study performed on the swept Hiemenz flow for conditions $R e=400, \omega=0$, showing sensitivity of radius on spanwise wavelength $\lambda$.

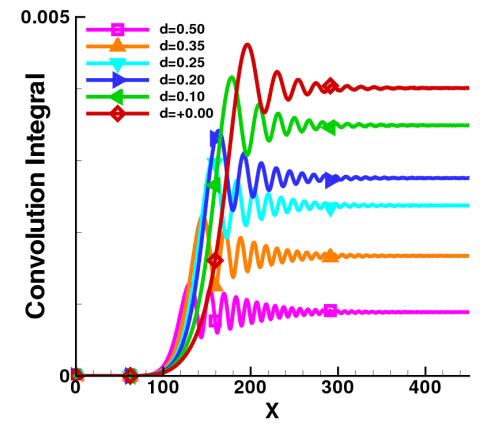

(a) HLNS: Positive Detuning.

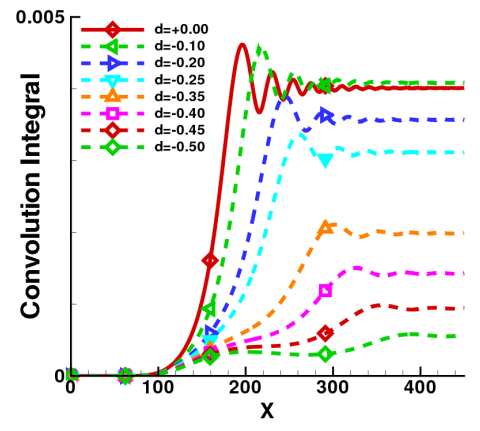

(b) HLNS: Negative Detuning.

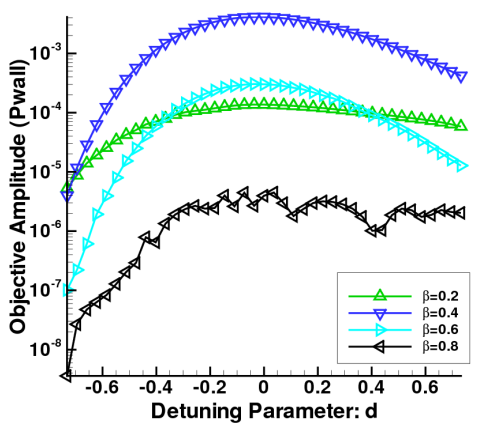

(c) Detuning for variable $\beta$

Figure 5. HLNS: Detuning study performed on the swept Hiemenz flow for conditions $R e=400, \beta=0.4$ are shown in Figs. (a) and (b). The detuning parameter is defined as $d=\left(\alpha_{w}-\alpha_{\text {neutral }}\right) / \alpha_{\text {neutral }}$ where $\alpha_{w}$ is the chordwise wavelength of the surface disturbance. Figure (c) shows a detuning study for the spanwise wavenumbers $\beta=0.2,0.4,0.6,0.8$.

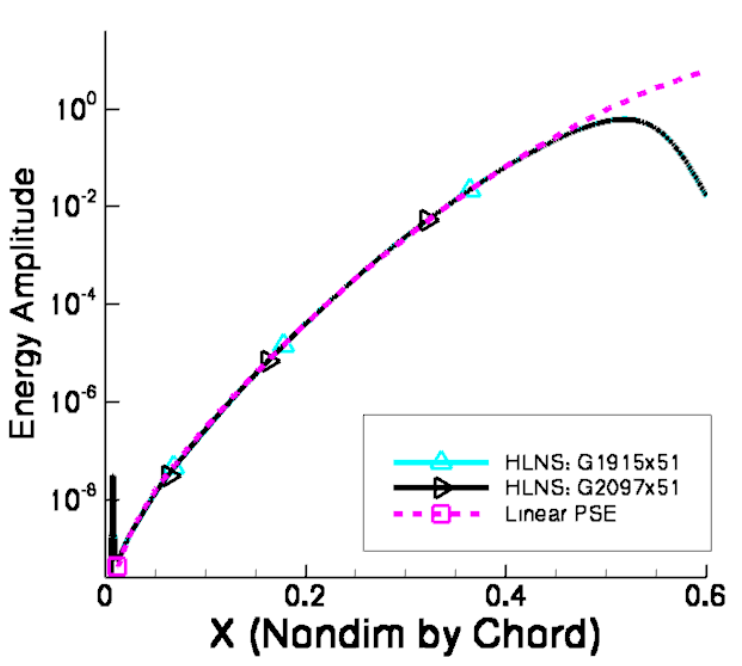

(a) Disturbance Energy Growth: Full

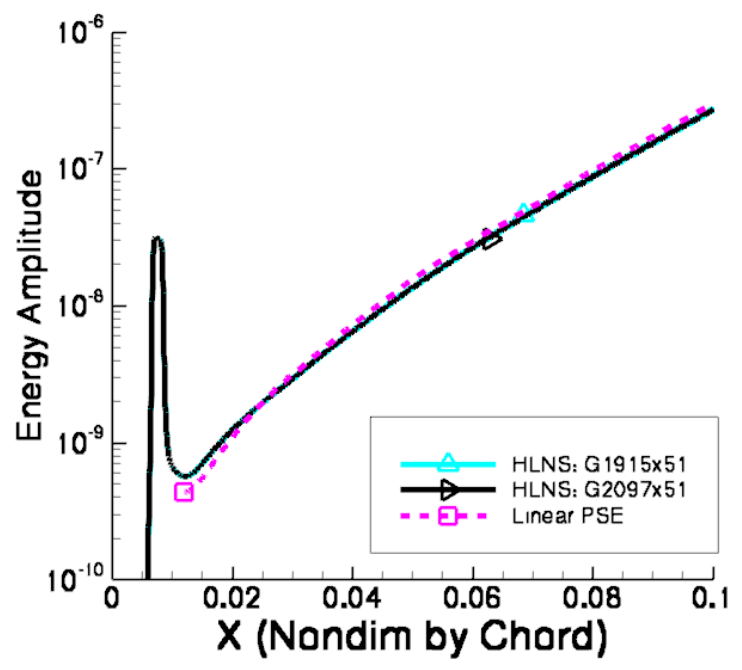

(b) Disturbance Energy Growth: Zoom

Figure 6. Growth of the disturbance energy with respect to chordwise position $x$, comparing linear PSE and HLNS. Flow conditions are $R e=7.15 \times 10^{6}, \beta=1915$ ( $4.5 \mathrm{~mm}$ wavelength) and $\omega=0$. Two grid resolutions are used for the HLNS simulations to assess convergence. 


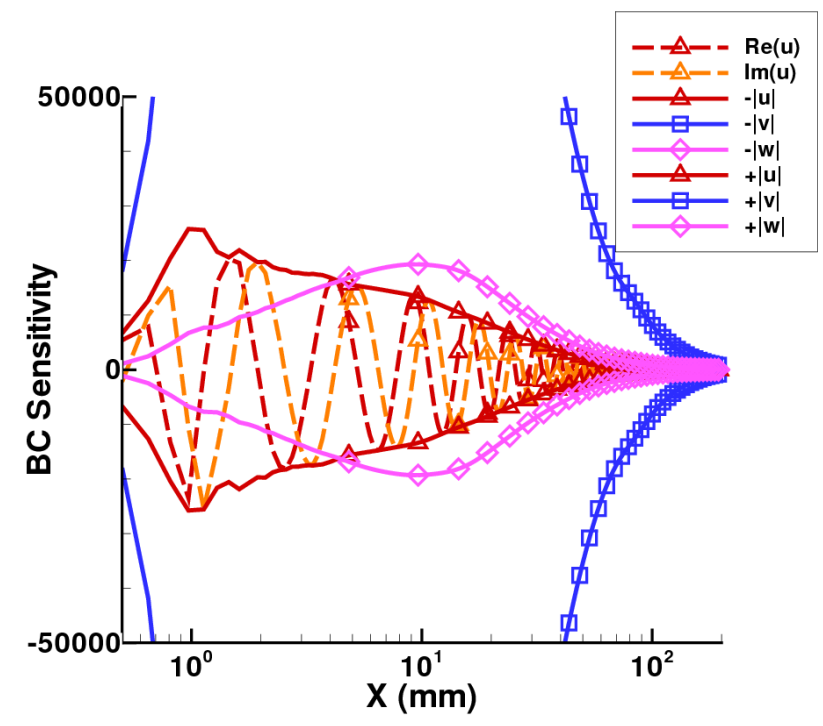

(a) Adjoint HLNS: $\Psi_{u}$ solution

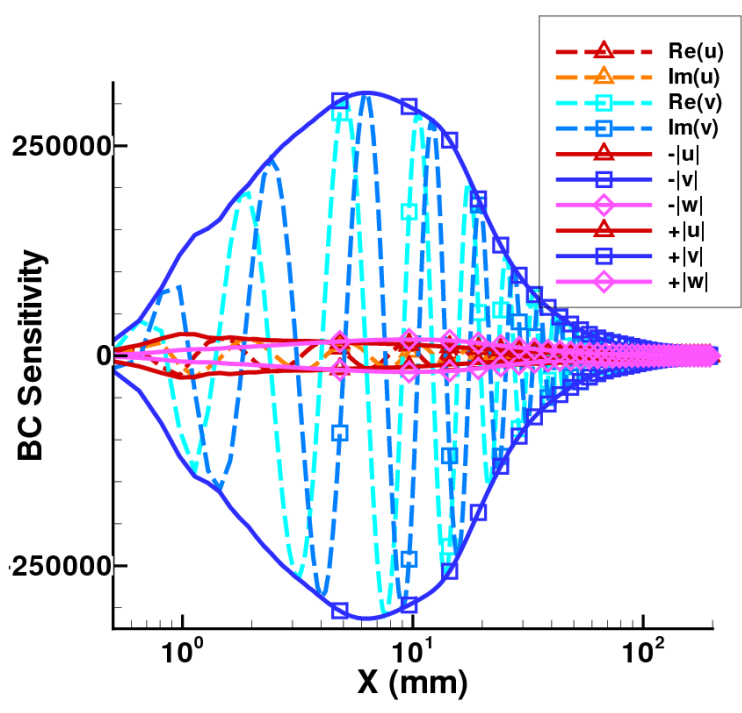

(b) Adjoint HLNS: $\Psi_{v}$ solution

Figure 7. Adjoint HLNS solutions for the SWIFT airfoil flow for conditions $R e=7.15 \times 10^{6}, \beta=0.0314(4.5 \mathrm{~mm}$ wavelength). The region of maximum sensitivity is approximately $10 \mathrm{~mm}$ and decreases rapidly downstream. The $\Psi_{v}$ is the most sensitive component.

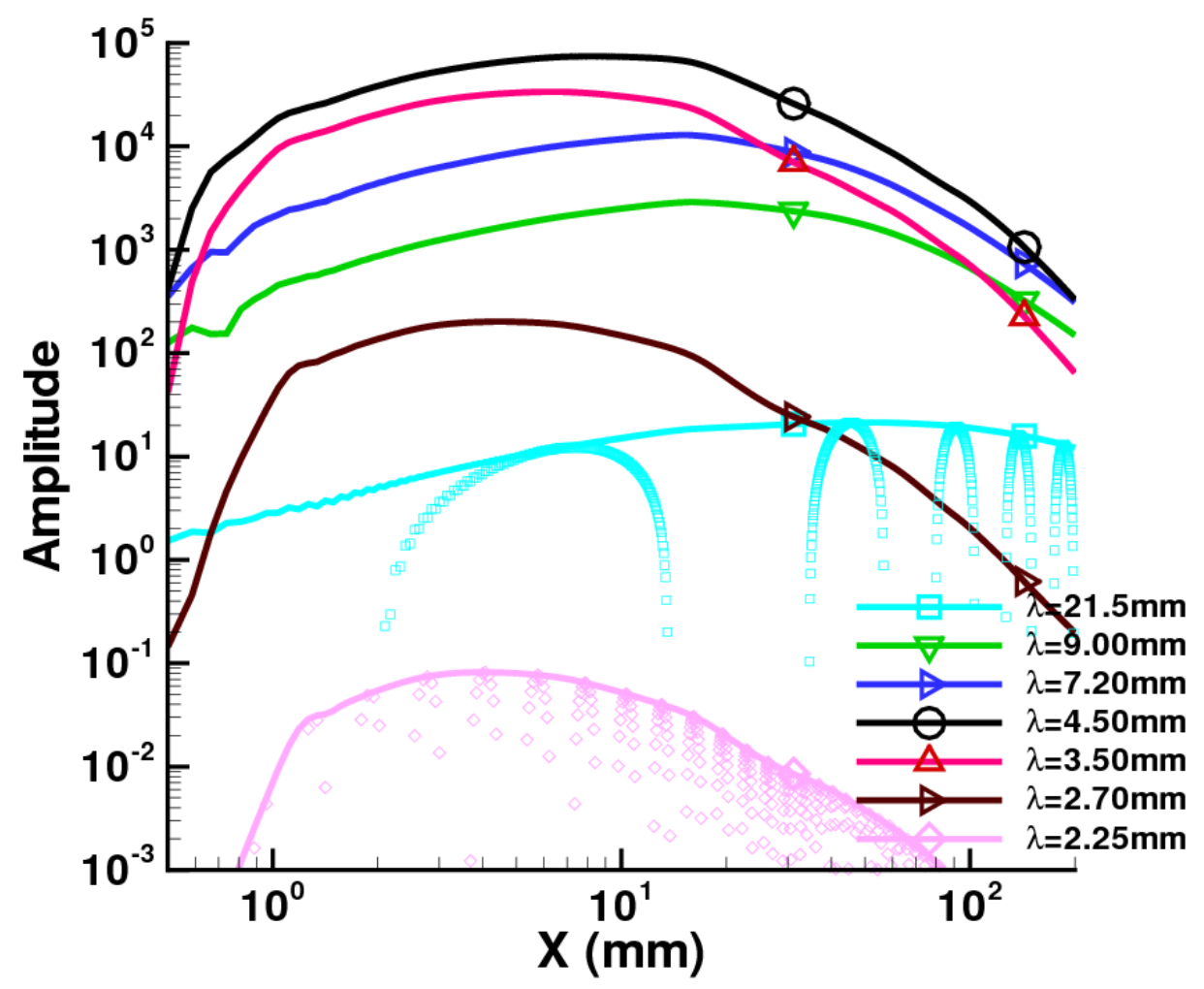

Figure 8. The $\left|\Psi_{v}\right|$ solution is presented for 7 spanwise wavelengths, centered about $4.5 \mathrm{~mm}$. The real component $R e\left[\Psi_{v}\right]$ is also included for the shortest and longest wavelengths, to show the dependency of (chordwise) wavelength on position $x$. 


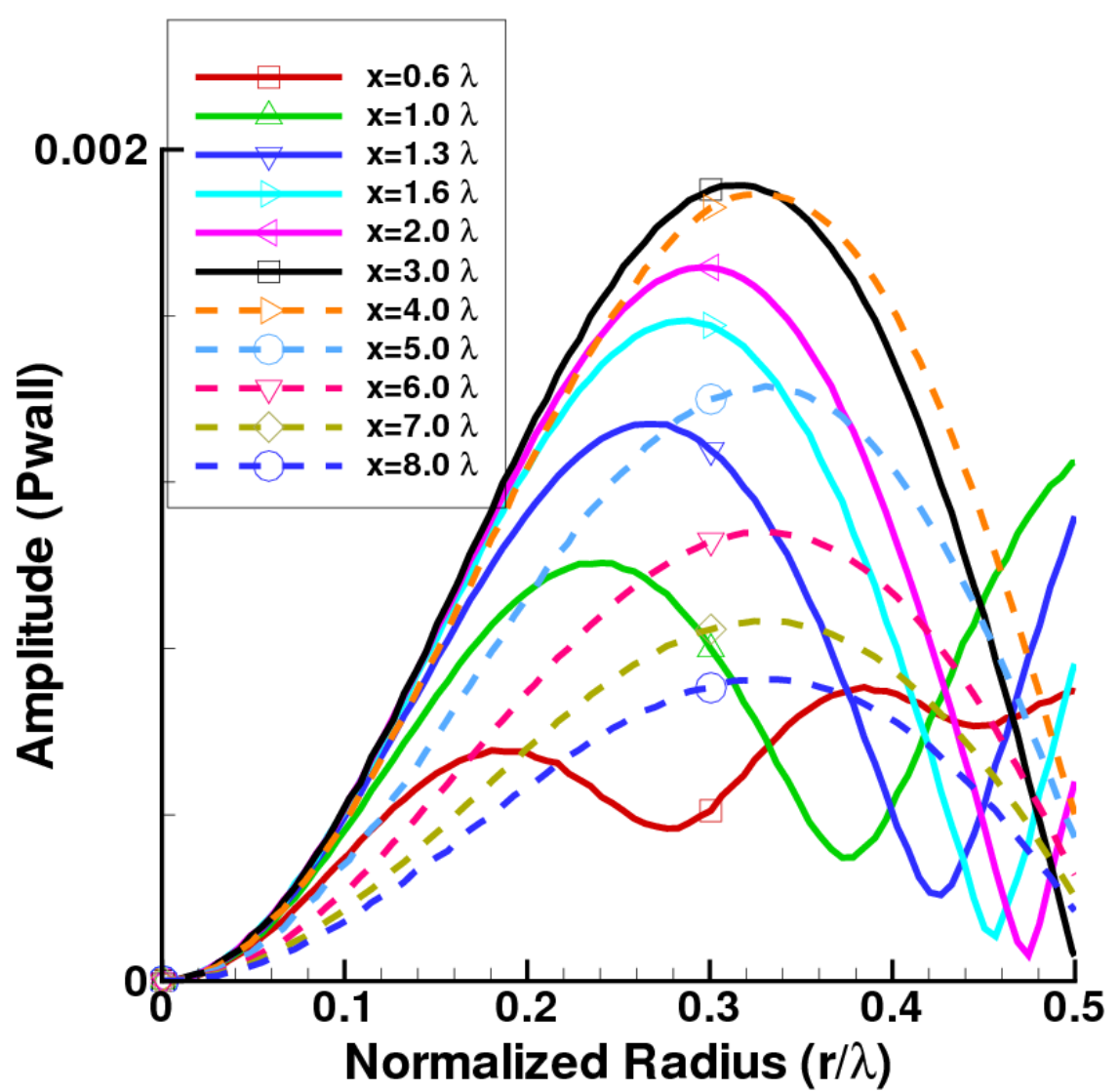

Figure 9. The sensitivity to DRE radius and location is examined for the SWIFT airfoil flow for conditions $R e=7.15 \times 10^{6}$, $\beta=1915$ ( $4.5 \mathrm{~mm}$ wavelength). The chordwise region of maximum sensitivity is about 3 wavelengths, and coincides approximately with the neutral location.

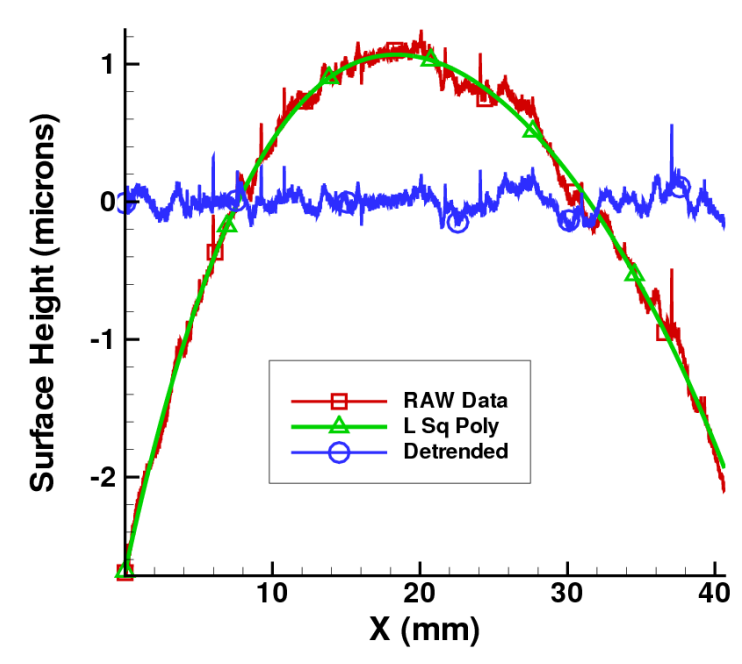

(a) Raw and Detrended Surface Data

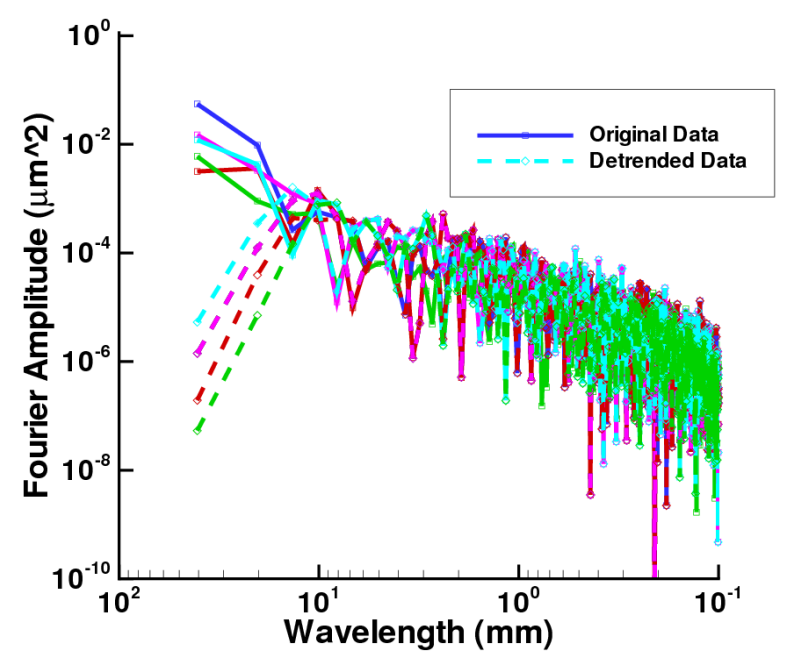

(b) Corresponding Fourier Spectrums

Figure 10. Figure (a) demonstrates the process of detrending on a taped a dataset. First the raw data are fitted with a low-order polynomial, which is then subtracted from the original data to yield a detrended dataset. Figure (b) shows the impact of detrending on the Fourier coefficients of a polished dataset. Detrending primarily affects the long wavelength modes. 


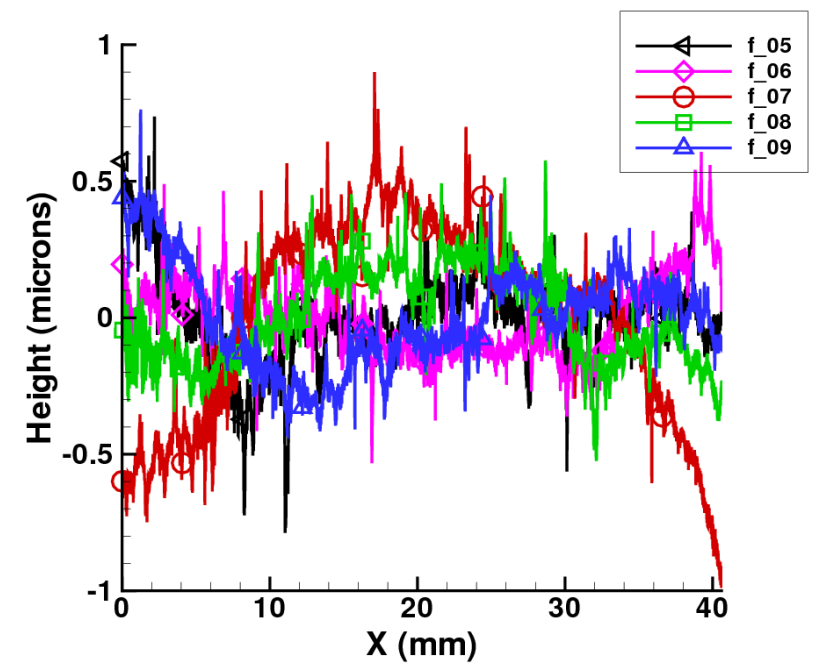

(a) Raw Polished Surface Data

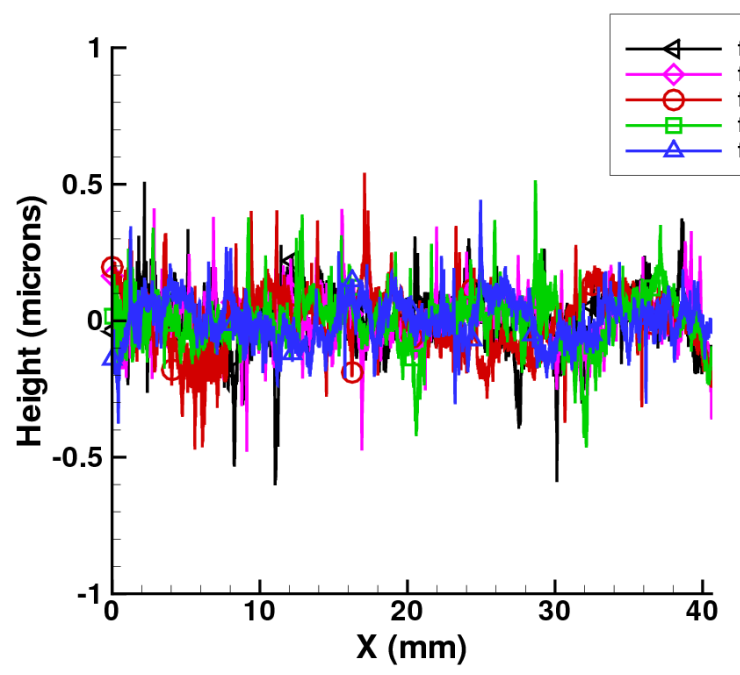

(b) Detrended Polished Surface Data

Figure 11. Line data for the five surfaces in the polished group. Figure (a) shows the raw surface data, while Fig. (b) shows the data after detrending.

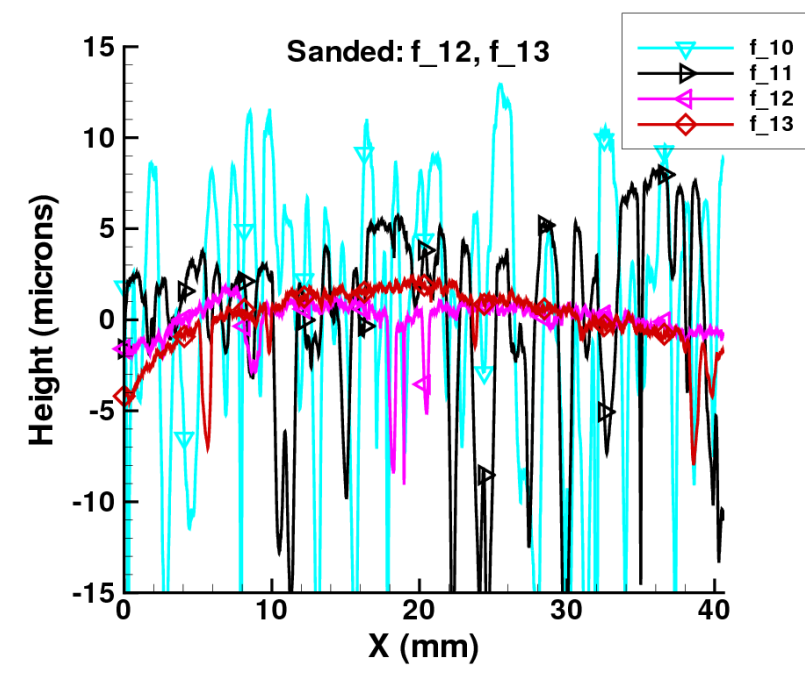

(a) Raw Painted Surface Data

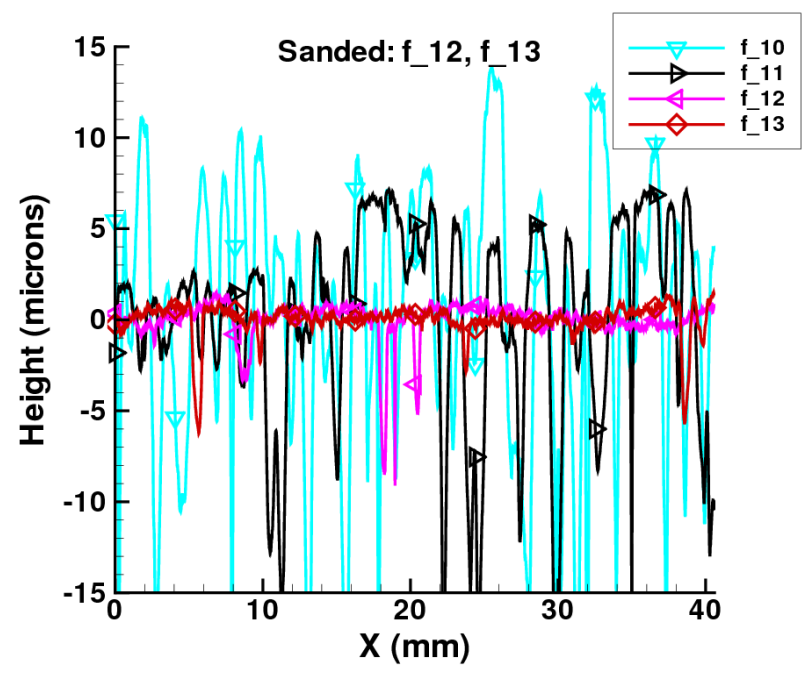

(b) Detrended Painted Surface Data

Figure 12. Line data for the two surfaces in the painted group, and three surfaces of the painted/sanded group. Figure (a) shows the raw surface data, while Fig. (b) shows the data after detrending. 


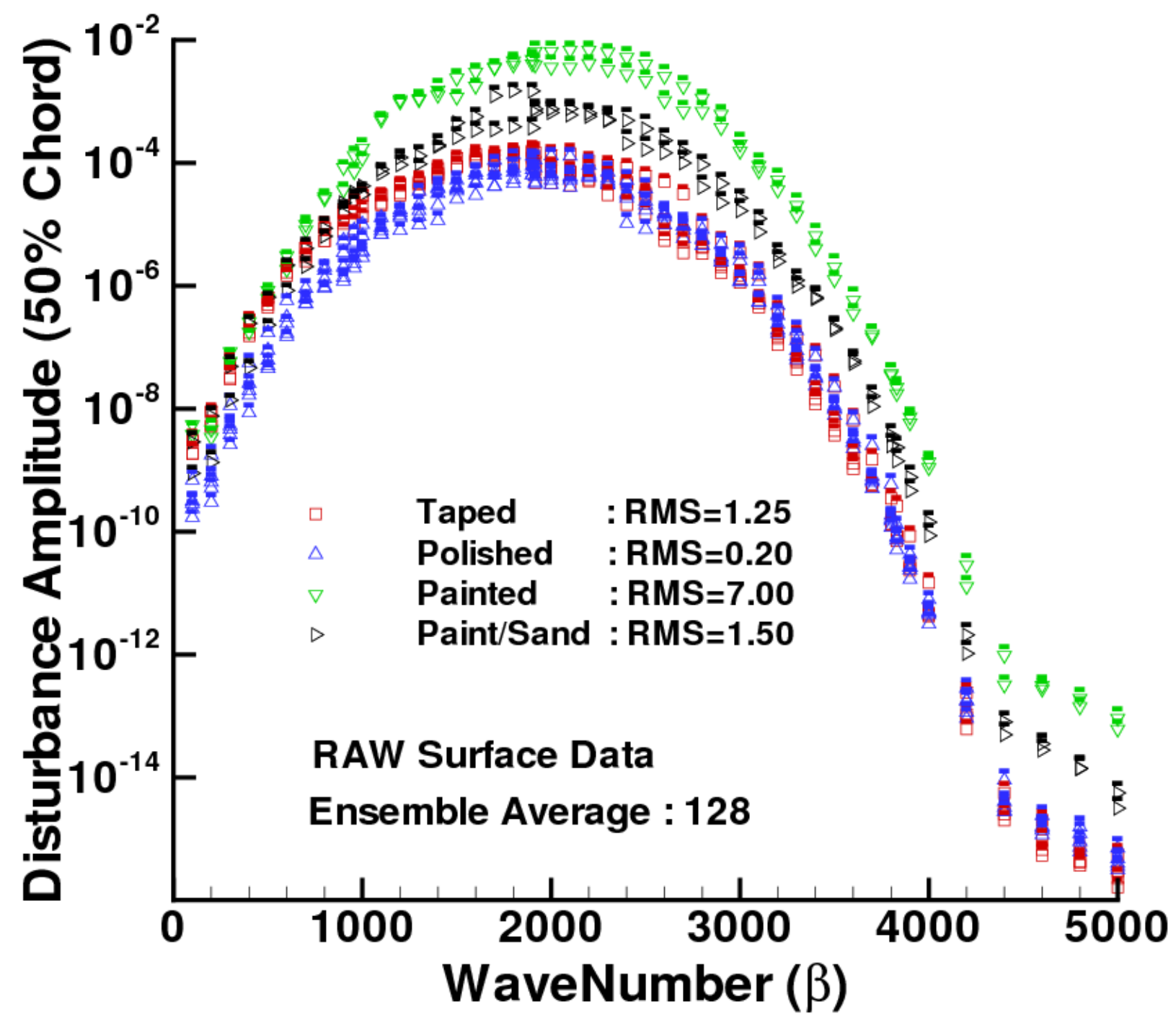

Figure 13. The Adjoint HLNS formulation is used to simulate the crossflow disturbance response to four classes of surface roughness: Taped, polished, painted and painted/sanded. $R e=7.15 \times 10^{6}, \omega=0$. The adjoint cost functional is placed at $50 \%$ chord. Raw surface data are used in the study. 


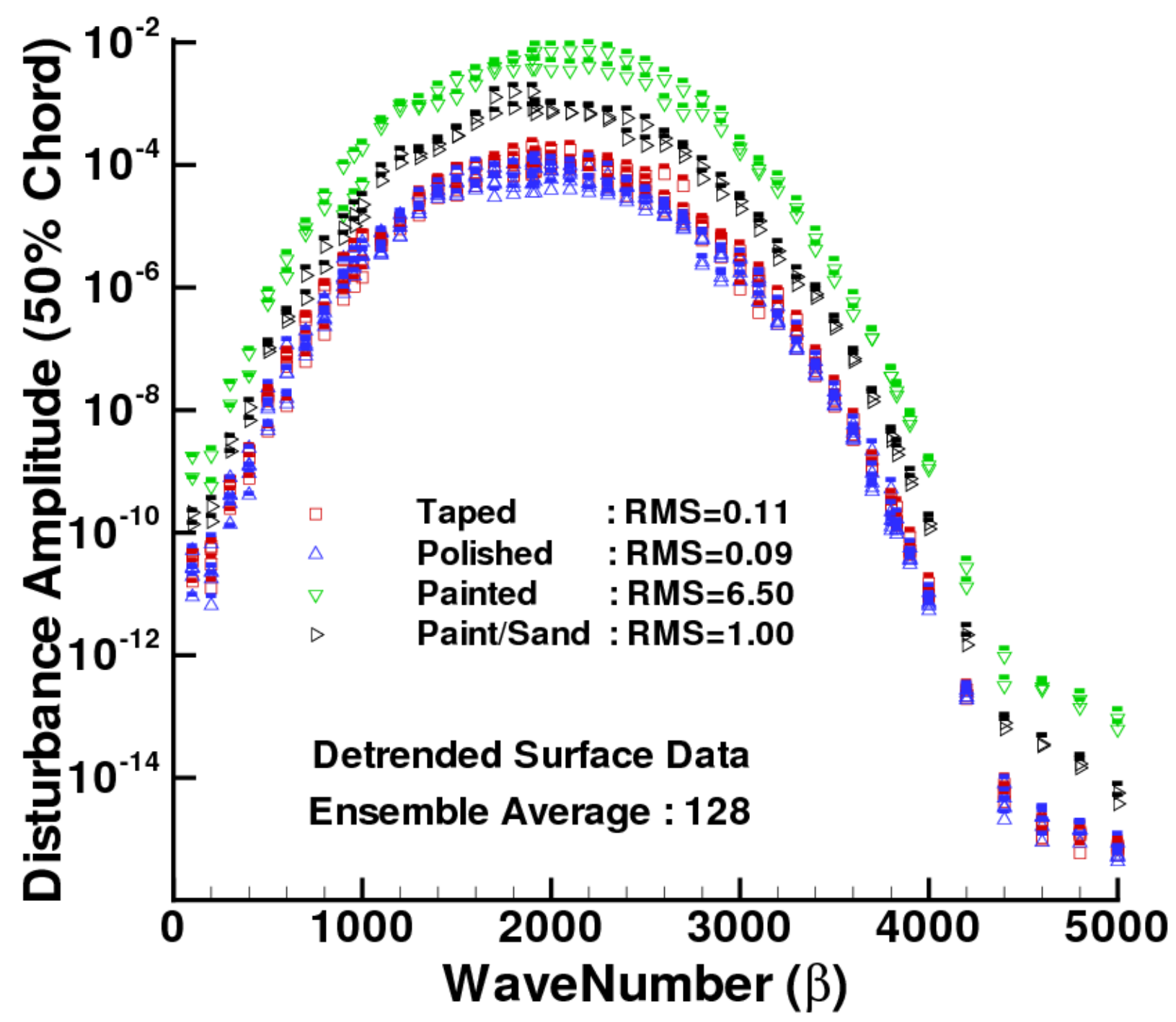

Figure 14. The Adjoint HLNS formulation is used to simulate the crossflow disturbance response to four classes of surface roughness: Taped, polished, painted and painted/sanded. $R e=7.15 \times 10^{6}, \omega=0$. The adjoint cost functional is placed at $50 \%$ chord. Detrended surface data are used in the study. 